\title{
Size and properties of the NLR in the Seyfert-2 galaxy NGC 1386^
}

\author{
N. Bennert ${ }^{1}$, B. Jungwiert ${ }^{2,3}$, S. Komossa ${ }^{4}$, M. Haas ${ }^{1}$, and R. Chini ${ }^{1}$ \\ 1 Astronomisches Institut Ruhr-Universität Bochum, Universitätsstrasse 150, 44780 Bochum, Germany \\ e-mail: [nbennert; haas; chini] @astro.rub.de \\ 2 Astronomical Institute, Academy of Sciences of the Czech Republic, Boční II 1401, 14131 Prague 4, Czech Republic \\ e-mail: bruno@ig.cas.cz \\ 3 CRAL-Observatoire de Lyon, 9 avenue Charles André, 69561 Saint-Genis-Laval Cedex, France \\ 4 Max-Planck Institut für extraterrestrische Physik, Giessenbachstrasse 1, 85748 Garching, Germany \\ e-mail: skomossa@xray.mpe.mpg.de
}

Received 3 June 2005 / Accepted 3 October 2005

\section{ABSTRACT}

We study the narrow-line region (NLR) of the Seyfert-2 galaxy NGC 1386 by means of long-slit spectroscopy obtained with FORS1 at the VLT. We use the galaxy itself for subtracting the stellar template, applying reddening corrections to fit the stellar template to the spectra of the NLR. The continuum gets steadily redder towards the nucleus. The spatial distribution of the reddening derived from the Balmer decrement differs from the continuum reddening, indicating dust within the NLR with a varying column density along the line of sight. Using spatially resolved spectral diagnostics, we find a transition between central line ratios falling into the AGN regime and outer ones in the H II-region regime, occuring at a radius of $\sim 6^{\prime \prime}(310 \mathrm{pc}$ ) in all three diagnostic diagrams. Applying CLOUDY photoionisation models, we show that the observed distinction between HII-like and AGN-like ratios in NGC 1386 represents a true difference in ionisation source and cannot be explained by variations of physical parameters such as ionisation parameter, electron density or metallicity. We interpret it as a real border between the NLR, i.e. the central AGN-photoionised region and surrounding H II regions. We derive surface brightness, electron density, and ionisation parameter as a function of distance from the nucleus. Both the electron density and the ionisation parameter decrease with radius. We discuss the consequences of these observations for the interpretation of the empirical NLR size-luminosity relation. In the outer part of the NLR, we find evidence for shocks, resulting in a secondary peak of the electron-density and ionisation-parameter distribution north of the nucleus. We compare the NLR velocity curve with the stellar one and discuss the differences.

Key words. galaxies: active - galaxies: individual: NGC 1386 - galaxies: nuclei - galaxies: Seyfert

\section{Introduction}

\subsection{The narrow-line region in active galaxies}

The narrow-line region (NLR) in active galactic nuclei (AGNs) is thought to be ionised by the luminous central engine, most likely an accreting supermassive black hole (BH). The narrow emission-lines are used in essentially all optical surveys to find and classify AGNs. Emission-line diagnostic provides a powerful tool to study the size and properties of the NLR. However, until today, even basic issues remain open: For example, what determines the NLR size and structure? What are the physical conditions within the NLR such as electron density and ionisation parameter and do they vary with distance from the nucleus?

Recently, a relation between NLR size and [O III] $\lambda 5007 \AA$ (hereafter [O III]) luminosity has been discovered from HST narrow-band images of seven radio-quiet PG

^ Based on observations made with ESO Telescopes at the Cerro Paranal Observatory under programme ID 72.B-0144. quasars (Bennert et al. 2002). However, the slope is discussed controversially: While Bennert et al. (2002) find $R \propto L_{[\mathrm{OIII}]}^{0.5}$, suggesting a self-regulating mechanism that determines the size to scale with the ionisation parameter, Schmitt et al. (2003b) report a relation of $R \propto L_{[\mathrm{OIII}]}^{0.33}$ for their sample of 60 Seyfert galaxies imaged with HST. Such a slope is expected for gas ionised by a central source (Strömgren) in the case of constant density.

Studying the [O III] emission alone as a tracer of the NLR size and structure has the major drawback that this emission can be contaminated by contributions from starbursts, shockionised gas or tidal tails, resulting in an apparent increase of the NLR. In addition, different sensitivities can lead to different size measurements: When comparing groundbased [O III] images of Seyfert galaxies from Mulchaey et al. (1996a) with the HST snapshot survey of Schmitt et al. (2003a), the latter reveal, on average, six times smaller NLR sizes, probably due to the 15 to 20 times lower sensitivity. These considerations question the definition "NLR size" from [O III] imaging alone. 
Long-slit spectroscopy is a valuable alternative approach as it can directly probe the size in terms of AGN photoionisation and discriminate the contribution of stellar or shock ionisation. In addition, several physical properties of the NLR such as the electron density and the ionisation parameter can be measured as a function of distance from the nucleus. Thus, it allows e.g. to probe the constant density needed to explain the 0.33 slope of the NLR size-luminosity relation. These parameters are also of general interest as they are important input parameters of photoionisation models of the NLR.

A well suited object for such an approach is the nearby $\left(v_{\text {hel }}=868 \pm 5 \mathrm{~km} \mathrm{~s}^{-1}, \mathrm{NED}^{1}\right)$ Seyfert-2 galaxy NGC 1386, allowing for a detailed study of the extended NLR down to small spatial scales $\left(1^{\prime \prime} \simeq 52 \mathrm{pc}\right)$.

We here describe long-slit spectroscopic observations obtained with FORS1 ${ }^{2}$ at the VLT $^{3}$ and the methods used to determine the "real" NLR size as well as physical conditions within the NLR. These methods were applied to a larger sample of Seyfert galaxies, revealing similar results as those for NGC 1386 which will be discussed in a subsequent paper.

\subsection{NGC 1386}

NGC 1386 is one of the nearest known Seyfert galaxies $(D \sim$ $11 \mathrm{Mpc}$ for $v_{3 \mathrm{~K}}=774 \mathrm{~km} \mathrm{~s}^{-1}$ and $H_{0}=71 \mathrm{~km} \mathrm{~s}^{-1} \mathrm{Mpc}^{-1} ; 1^{\prime \prime} \sim$ $52 \mathrm{pc})$.

Due to its relatively high inclination $\left(i \simeq 71^{\circ}\right)$, the morphological galaxy type has been a matter of debate: While several authors favour S0, an Sa classification is assumed by Weaver et al. (1991). Malkan et al. (1998) give a morphological type of $\mathrm{Sb} / \mathrm{c}$ based on HST imaging, noting that they possibly have missed bars on larger spatial scales. In RC3 (De Vaucouleurs et al. 1991), NGC 1386 is indeed classified as barred SB0 galaxy. Also NED gives $\mathrm{SB}(\mathrm{s}) 0+$ as morphological classification.

NGC 1386 has been investigated by various authors. We here summarise the most important results with respect to our study.

Weaver et al. (1991) study the extended NLR by means of groundbased imaging as well as long-slit spectroscopy with multiple slit positions. They show a $2 \mathrm{D}$ coverage of the inner $\mathrm{kpc}$ of this galaxy and discuss the overall morphology, velocity field, electron-density distribution, and ionisation structure. They use the galaxy itself as stellar template to correct for underlying absorption lines. Using one diagnostic line-ratio diagram $([\mathrm{O}$ III $] / \mathrm{H} \beta$ versus $[\mathrm{N}$ II $] \lambda 6583 \AA / \mathrm{H} \alpha$ ), they determine the NLR (i.e. the AGN powered region) to extend $\sim 6^{\prime \prime}$ to the north and south of the nucleus while emission lines further out can be attributed to $\mathrm{H}$ II regions. They find an electron density which is decreasing with radius. The velocity field is interpreted in terms of a combination of a normally rotating component and a component undergoing high-velocity infall or outflow.

Mauder et al. (1992) resolve the NLR down to a linear scale of 0 ! 3 ( $\simeq 15 \mathrm{pc})$ using speckle interferometry and detect

\footnotetext{
${ }^{1}$ NASA/IPAC Extragalactic Database.

2 FOcal Reducer/low dispersion Spectrograph.

${ }^{3}$ Very Large Telescope, Cerro Paranal, Chile (ESO).
}

individual NLR clouds on these scales. They argue that the ionising radiation must be absorbed on scales $<15 \mathrm{pc}$ in a clumpy structure of the NLR.

Tsvetanov \& Petrosian (1995) give positions and fluxes of $44 \mathrm{H} \mathrm{II}$ regions distributed in a ring seen in their continuumsubtracted $\mathrm{H} \alpha+[\mathrm{N} \mathrm{II}] \lambda \lambda 6548,6583 \AA$ (hereafter [N II]) image. The distances of the H II regions are $\sim 6-12^{\prime \prime}$ from the nucleus at a position angle (PA) of the major axis of the ring of $25^{\circ}$, comparable to the PA of the major axis of the galaxy $\left[25^{\circ}\right.$; RC3 (De Vaucouleurs et al. 1991)].

Ferruit et al. (2000) present HST images of the [O III] and $\mathrm{H} \alpha+[\mathrm{N} \mathrm{II}]$ emission. The images show the presence of very strong dust features, especially on the north-west side of the galaxy and in the nuclear regions. They find gradients in the $[\mathrm{O} \mathrm{III}] /([\mathrm{N} \mathrm{II}]+\mathrm{H} \alpha)$ ratio which at least partly cannot be due to dust lanes but may correspond to a real transverse change in the excitation conditions of the ionised gas. Ferruit et al. (2000) report a faint inclined ring of $\mathrm{H}$ II-region emission extending out to $\sim 12^{\prime \prime}$ from the nucleus with a PA of $25^{\circ}$, in agreement with the results from Tsvetanov \& Petrosian (1995). The [O III] image of Ferruit et al. (2000) is included in the study of Schmitt et al. (2003a) and presented here in Fig. 1 with the slit position of our observation overlayed $\left(\mathrm{PA}_{[\mathrm{OIII}]}=5^{\circ}\right)$. It traces the direction of the maximum extent in [O III] which is displaced by $20^{\circ}$ from the host galaxy major axis $\left(\mathrm{PA}_{\text {galaxy }}=25^{\circ}\right)$ and by $\sim 15^{\circ}$ from the extended radio emission $\left[\mathrm{PA}_{\text {radio }}=170^{\circ}\right.$, Nagar et al. 1999].

Rossa et al. (2000) and Schulz \& Henkel (2003) study the kinematics of the inner emission-line region in detail. Both find evidence for a regular velocity field showing signs of rotation. The line profiles show pronounced substructure suggestive of non-circular motions like locally expanding gas systems. Schulz \& Henkel (2003) suggest a near-edge-on warped rotating spiral disk as traced by $\mathrm{H} \alpha$ with a central velocity gradient corresponding to a Keplerian mass estimate of $\sim 5 \times 10^{9} \mathrm{M}_{\odot}$ inside $0.8 \mathrm{kpc}$. Like Mauder et al. (1992), Rossa et al. (2000) detect several individual NLR components.

NGC 1386 is also included in the sample of 18 Seyfert-2 galaxies studied with long-slit spectroscopy by Fraquelli et al. (2003). They present reddening and surface-brightness distributions as well as a decreasing electron density observed along a PA of $169^{\circ}$. However, their data have a significant lower signal-to-noise ratio $(\mathrm{S} / \mathrm{N})$ than our data and, moreover, they did not take into account the underlying absorption owing to the contribution of the stellar population which we show to be important.

\section{Observations}

The high S/N long-slit spectra of NGC 1386 were obtained using FORS1 attached to the Cassegrain focus of UT1 at the VLT on the 25th of February 2004. Observations were made in the spectral range 3050-8300 $\AA$ through the nucleus of NGC 1386 with exposure times of $1800 \mathrm{~s}$ (to study the emission lines far from the nucleus) and $30 \mathrm{~s}$ (to gain the central emission lines which were saturated in the $1800 \mathrm{~s}$ exposure) with a typical seeing of $\sim 1^{\prime \prime}$, under the use of the atmospheric dispersion corrector. The detector used is a $2048 \times 2048$ pixels Tektronix 


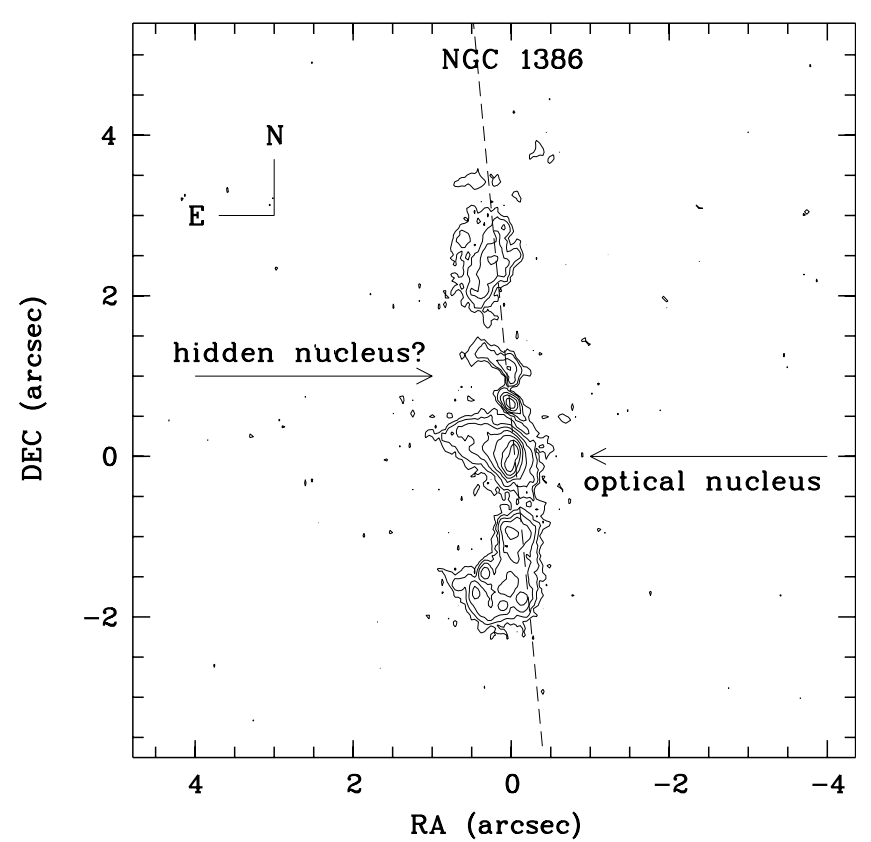

Fig. 1. [O III] continuum-subtracted image of NGC 1386 taken with HST, kindly provided by Henrique Schmitt. The contours start at the $3 \sigma$ level above the background $\left(2.1 \times 10^{-14} \mathrm{erg} \mathrm{cm}^{-2} \mathrm{~s}^{-1} \mathrm{arcsec}^{-2}\right)$ and increase in powers of 2 times $3 \sigma\left(3 \sigma \times 2^{n}\right)$. The exposure time is $800 \mathrm{~s}$. The pixel size is $00^{\prime} 0455$. The [O III] emission was centered on the optical nucleus determined from the continuum image by Schmitt et al. (2003a). The velocity curve suggests a hidden nucleus at $\sim 1^{\prime \prime}$ north of the optical nucleus. The slit position of our observations at PA = $5^{\circ}$, tracing the major axis of the [O III] extension, is indicated by the dashed line.

CCD with $24 \mu \mathrm{m}$ wide square pixels. The spatial resolution element is $0.2 \mathrm{pix}^{-1}$. The slit width corresponds to 0.7 on the sky projecting to a spectral resolution of $\sim 8 \AA\left(\sim 450 \mathrm{~km} \mathrm{~s}^{-1}\right)$ as is confirmed by the full-width-at-half-maximum (FWHM) of wavelength calibration lines and the [O I] $\lambda 5577 \AA$ night-sky line. The long slit was orientated along the PA of the maximum extent of the high excitation gas observed in the [O III] image $\left(\mathrm{PA}_{[\mathrm{OIII}]}=+5^{\circ} ;\right.$ Fig. 1$)$ and corresponds to 6.8 in the sky.

\section{Reduction and analysis}

\subsection{Data reduction}

Standard reduction including bias subtraction, flat-field correction, and cosmic-ray rejection was performed using the ESO MIDAS 4 software (version Nov. 99). Night-sky spectra at $1^{\prime}-3^{\prime}$ distance on both sides of any notable galaxy emission were interpolated in the region of the galactic spectrum and subtracted. The spectra were rebinned to a scale of $2.65 \AA \mathrm{pix}^{-1}$ during wavelength calibration. The curve of Tüg (1997) was used to correct for atmospheric extinction. The spectra were flux calibrated using the standard star CD$32^{\circ} 9927$. Foreground Milky Way reddening was corrected using values from Schlegel et al. (1998) as listed in NED $\left(E_{(B-V), G}\right.$ $=0.012 \mathrm{mag}$ ) and the extinction law from Savage et al. (1979). Forbidden-line wavelengths were taken from Bowen (1960). A

\footnotetext{
${ }^{4}$ Munich Image Data Analysis System, trade mark of ESO.
}

heliocentric correction of $-28.8 \mathrm{~km} \mathrm{~s}^{-1}$ was added to the observed velocities.

In the spatial direction perpendicular to the dispersion axis, five pixel rows were averaged from the frames cleaned in this way according to the seeing to enhance the $\mathrm{S} / \mathrm{N}$ without loosing any spatial information. Thus, each resulting extracted spectrum of the spatially resolved emission corresponds to $1^{\prime \prime}$ and 0.7 along and perpendicular to the slit direction, respectively. We choose the spectrum with the maximum intensity of the continuum as "photometrical center" ("zero" on the spatial scale). It coincides with the highest emission-line fluxes in $\mathrm{H} \alpha$ and [O III]. In the following, we also refer to it as "central spectrum".

\subsection{Subtracting the stellar population}

Starlight constitutes a substantial fraction of light gathered in the optical spectra of AGNs, particularly in low-luminosity objects such as LINERs and Seyfert galaxies. The emission-line fluxes are affected by underlying absorption lines which can be large especially in the case of $\mathrm{H} \beta$. As a consequence, starlight can have a great influence on the determination of the reddening and the AGN diagnostic line ratio $\mathrm{H} \beta /[\mathrm{O}$ III]. Thus, removing the contribution of the stellar population is one of the first and most critical steps in the analysis of AGN emission-line spectra. The most widely used technique for starlight subtraction makes use of an appropriately chosen spectrum of a normal galaxy. However, the stellar populations in the inner regions of AGNs present a variety of characteristics (Cid Fernandes et al. 1998), giving rise to the need of a library with a large diversity of stellar populations. Alternatively, the shape and strength of the starlight component can be estimated by means of stellar population synthesis techniques, following the work pioneered by Bica (1988). The classification refers to the library of synthetic spectra of Bica (1988), i.e. templates composed of different percentages of star cluster spectra of several ages and metallicities. An S3 template, for example, contains only the contribution of stars older than 10 Gyr. In S4, 95\% of the light at $5870 \AA$ comes from old stars and $5 \%$ from young and intermediate age ones. In S5, the contribution is $85 \%$ from old stars and $15 \%$ from young and intermediate age ones, etc. Thus, a low number "Sx" indicates a rather old stellar population, a high number a young one. This method, however, involves several assumptions of stellar modeling and does not necessarily yield an unambiguous solution.

Here, we applied the straightforward approach of using the galaxy itself as starlight template which should give the best representation of the stellar population. The high-sensitivity spectrum allows us to average several pixel rows with high $\mathrm{S} / \mathrm{N}$ in the outer part of the galaxy spectrum. One assumption is that the stellar population does not vary much in the inner few arseconds, i.e. the bulge, as is confirmed by the close match of the stellar template and the absorption lines in all studied spectra. Furthermore, we assume that the contribution of the AGN featureless continuum is negligible to not overestimate the underlying absorption lines by scaling the template to the continuum in each spectrum. This seems to be justified for type-2 
AGNs: Cid Fernandes et al. (1998) find little or no dilution of the stellar lines by an underlying featureless continuum for most of the 20 Seyfert-2s in their sample, including NGC 1386. Weaver et al. (1991) apply a similar method of subtracting the stellar contribution in NGC 1386.

The stellar template was gained at a distance of $\sim 18^{\prime \prime}$ (940 pc) north from the nucleus, averaged over $5 "$ and median filtered along the spectral axis over three pixels to increase the $\mathrm{S} / \mathrm{N}$. The extracted region is both far enough from the central region to exclude any NLR emission, as well as near enough to still guarantee a comparable stellar population with high $\mathrm{S} / \mathrm{N}$. This assumption is strenghtened by the stellar population analysis carried out for NGC 1386 by Cid Fernandes et al. (1998): The equivalent widths at the nucleus are typical of an S3 template out to $10^{\prime \prime}$ where the stellar population resembles S4-S5 due to the presence of spiral arms. This coincides with the inclined ring of emission seen in the $\mathrm{H} \alpha+[\mathrm{N} \mathrm{II}]$ image by Ferruit et al. (2000). Further out, the stellar population is again S3, and we can thus use the template determined at $\sim 18^{\prime \prime}$ to fit the nuclear spectra out to $10^{\prime \prime}$. The resulting template spectrum is clearly dominated by absorption lines showing strong $\mathrm{Ca}$ II $\mathrm{H}+\mathrm{K}$ lines as well as the Balmer series (Fig. 2, middle spectrum).

The template was scaled to each row of the NLR spectrum by normalization in the red $(\sim 5400-5700 \AA)$, justified by the fact that the slope at $\lambda \geq 5400 \AA$ does not change significantly for different stellar populations (Bica et al. 1986). (Note that we also chose this range as it does not cover any strong NLR emission lines.) To allow for a possible reddening difference of the template and each NLR spectrum due to different dust amounts in different galactic regions, we applied a reddening correction to the template by fitting the continuum slope of the template to each NLR spectrum [MIDAS command "extinct/long" with extinction-law from Savage et al. (1979)]. This procedure was often necessary to avoid a template continuum exceeding that of the NLR continuum at some wavelength after normalization.

In Fig. 3, we show the template with and without reddening correction with respect to the nucleus. The scaled (de-) reddened template was subtracted to gain the pure emissionline spectra. Finally, any remaining AGN continuum contribution was fit interactively by a continuum slope and subtracted (Fig. 2, lower spectrum).

\subsection{Emission-line fluxes and reddening}

The fluxes of the pure emission-line spectra were measured as a function of distance from the nucleus by integrating along a Gaussian fit to the line profile. The fit routine "fit/spec" (Rousset 1992) was used for this purpose. In the three central spectra (center $\pm 1^{\prime \prime}$ ), the strongest emission lines were saturated in the $1800 \mathrm{~s}$ exposure and all fluxes were derived from the 30 s nuclear exposure.

The uncertainties in deriving the fluxes were mostly caused by uncertainties in the placement of the continuum and were thus estimated as the product of the FWHM of the line and the root-mean square deviation of the local continuum fluxes. Gaussian error propagation was used to calculate the errors of

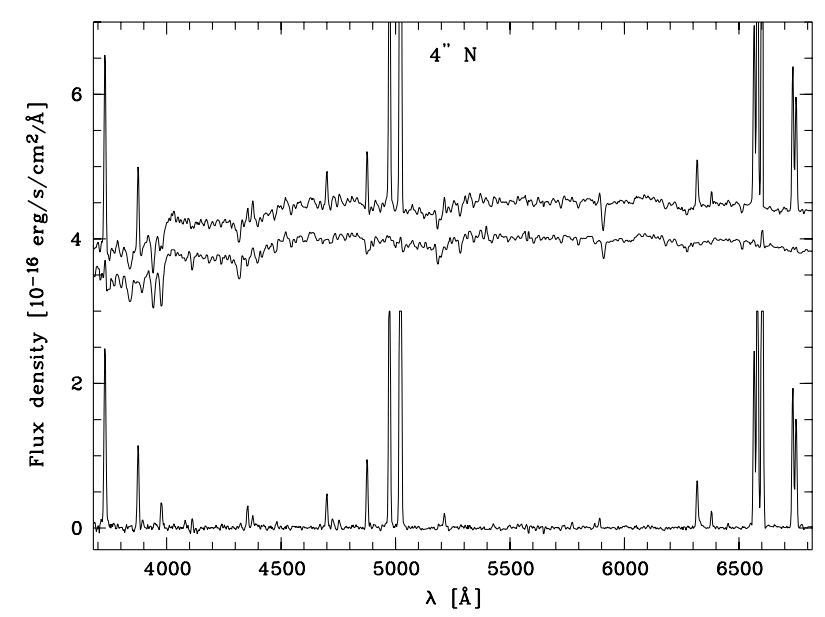

Fig. 2. Spectrum at a distance of $4 "$ north of the nucleus. The upper spectrum shows strong $\mathrm{Ca}$ II $\mathrm{H}+\mathrm{K}$ absorption lines and Balmer features. A template spectrum of the stellar contribution derived from the galaxy itself at $18^{\prime \prime}$ north of the nucleus was subtracted (middle spectrum). The difference spectrum is the lower one (with the stronger emission lines truncated). Both upper spectra are shifted by an arbitrary amount for comparison.

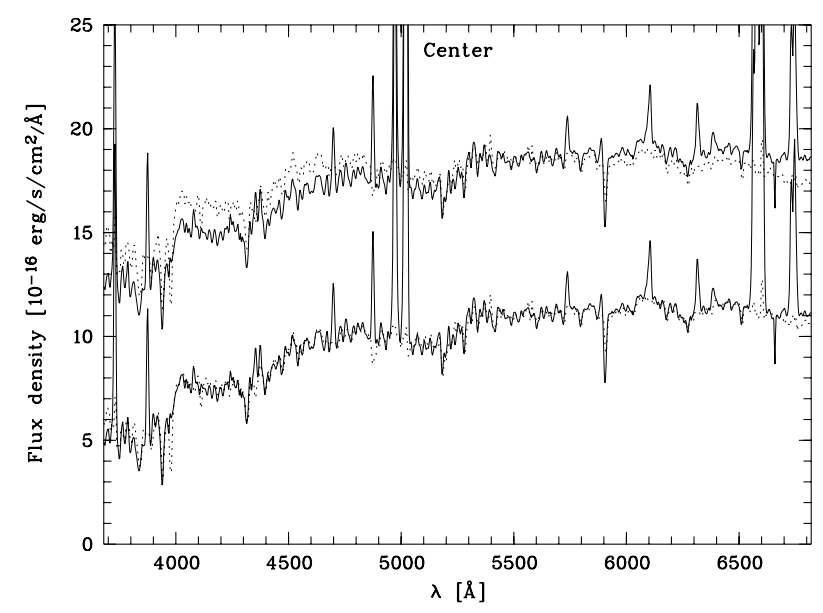

Fig. 3. Central spectrum of NGC 1386 with stellar template overlayed as dotted line. In the upper spectra, which were shifted by an arbitrary amount for comparison, the reddening of the nucleus with respect to the template can be clearly seen. In the lower spectra, a reddening correction was applied to the template to improve the match of the template and the continuum of the nuclear spectrum.

subsequent parameters such as line ratios, ionisation parameter, etc. The resulting erros are in the range of $\sim 1-15 \%$. Note that we did not take into account uncertainties from stellar absorption correction and the quality of the Gaussian fits which was very good given the low spectral resolution of our data.

The spectra were dereddened using the recombination value for the intensity ratio $\mathrm{H} \alpha / \mathrm{H} \beta=2.87$ (a typical value for $T=10000 \mathrm{~K}$, Osterbrock (1989), Table 4.2) and an average reddening curve (Osterbrock (1989), Table 7.2). Note that in the following, only those spectra are used which have emissionline fluxes exceeding the $\mathrm{S} / \mathrm{N}$ ratio of 3 . 
Table 1. Observed and reddening-corrected line-intensity ratios relative to $\mathrm{H} \beta$ for the nuclear spectrum.

\begin{tabular}{lcc}
\hline \hline Line & $F_{\text {obs }}$ & $F_{\text {dered }}$ \\
\hline$[\mathrm{O}$ II $] \lambda 3727 \AA$ & $1.81 \pm 0.02$ & 2.63 \\
{$[\mathrm{Ne}$ III $] \lambda 3869 \AA$} & $0.77 \pm 0.04$ & 1.07 \\
$\mathrm{H} \epsilon+[\mathrm{Ne}$ III $] \lambda 3967 \AA$ & $0.48 \pm 0.05$ & 0.64 \\
{$[\mathrm{O}$ III $] \lambda 4363 \AA$} & $0.19 \pm 0.02$ & 0.23 \\
$\mathrm{He}$ II $\lambda 4686 \AA$ & $0.46 \pm 0.05$ & 0.48 \\
{$[\mathrm{O}$ III $] \lambda 5007 \AA$} & $11.34 \pm 0.1$ & 10.73 \\
{$[\mathrm{Fe}$ VII $] \lambda 5721 \AA$} & $0.29 \pm 0.04$ & 0.22 \\
{$[\mathrm{Fe}$ VII $] \lambda 6087 \AA$} & $0.44 \pm 0.07$ & 0.31 \\
{$[\mathrm{O}$ I $] \lambda 6300 \AA$} & $0.46 \pm 0.02$ & 0.3 \\
{$[\mathrm{Fe}] \lambda 6375 \AA$} & $0.07 \pm 0.01$ & 0.05 \\
$\mathrm{H} \alpha$ & $4.7 \pm 0.05$ & 2.87 \\
{$[\mathrm{~N}$ II $] \lambda 6583 \AA$} & $5.6 \pm 0.06$ & 3.41 \\
{$[\mathrm{~S} \mathrm{II}] \lambda 6716 \AA$} & $1.04 \pm 0.05$ & 0.62 \\
{$[\mathrm{~S} \mathrm{II}] \lambda 6731 \AA$} & $1.29 \pm 0.07$ & 0.77 \\
\hline
\end{tabular}

\section{Results and discussion}

While a spectrum of NGC 1386 at 4 " north of the nucleus as well as a nuclear spectrum were already presented in Figs. 2 and 3, Fig. 4 additionally shows representative spectra at 5 " and 8 " north of the nucleus to demonstrate the emission-line variations with distance from the nucleus.

In Table 1, we present both the observed and reddeningcorrected line-intensity ratios relative to $\mathrm{H} \beta$ from the nuclear spectrum (uncorrected for slit losses). For pairs of lines ([O III], $[\mathrm{OI}]$, and $[\mathrm{N} \mathrm{II}])$ with a fixed line ratio $(\sim 3: 1)$, only the brighter line is listed. The reddening-corrected $\mathrm{H} \beta$ luminosity is $(1.42 \pm$ $0.01) \times 10^{40} \mathrm{erg} \mathrm{s}^{-1}$.

From the $[\mathrm{O} \mathrm{III}](\lambda 4959 \AA+\lambda 5007 \AA) / \lambda 4363 \AA$ emissionline ratio, we determine the electron temperature $T_{\mathrm{e}}$ in the nuclear spectrum to $T_{\mathrm{e}}=15650 \pm 1500 \mathrm{~K}$.

\subsection{Reddening distribution}

Two different measures of the reddening distribution were derived: (i) the above mentioned reddening of the continuum slope in the central parts of NGC 1386 with respect to the template derived in the outer parts of the galaxy; (ii) the reddening distribution obtained from the recombination value for the intensity ratio $\mathrm{H} \alpha / \mathrm{H} \beta$. Both reddening distributions are shown in Fig. 5.

As already noted by Weaver et al. (1991), the continuum gets steadily redder towards the nucleus. The reddest continuum is found $1^{\prime \prime}$ north of the nucleus. We observe a significant drop 3-4" north of the nucleus not reported by Weaver et al. (1991). It can be directly attributed to a "blue" continuum seen $3^{\prime \prime}$ north of the nucleus in the $[F 547 M / F 791 W]$ color map by Ferruit et al. (2000). The blue continuum is associated with the northern tip of the central emission-line structure in the [O III] image (Fig. 1).

As the match between the absorption lines of the stellar template and those seen in the spectra is quite close, we believe that reddening by dust is the cause of the spatially varying
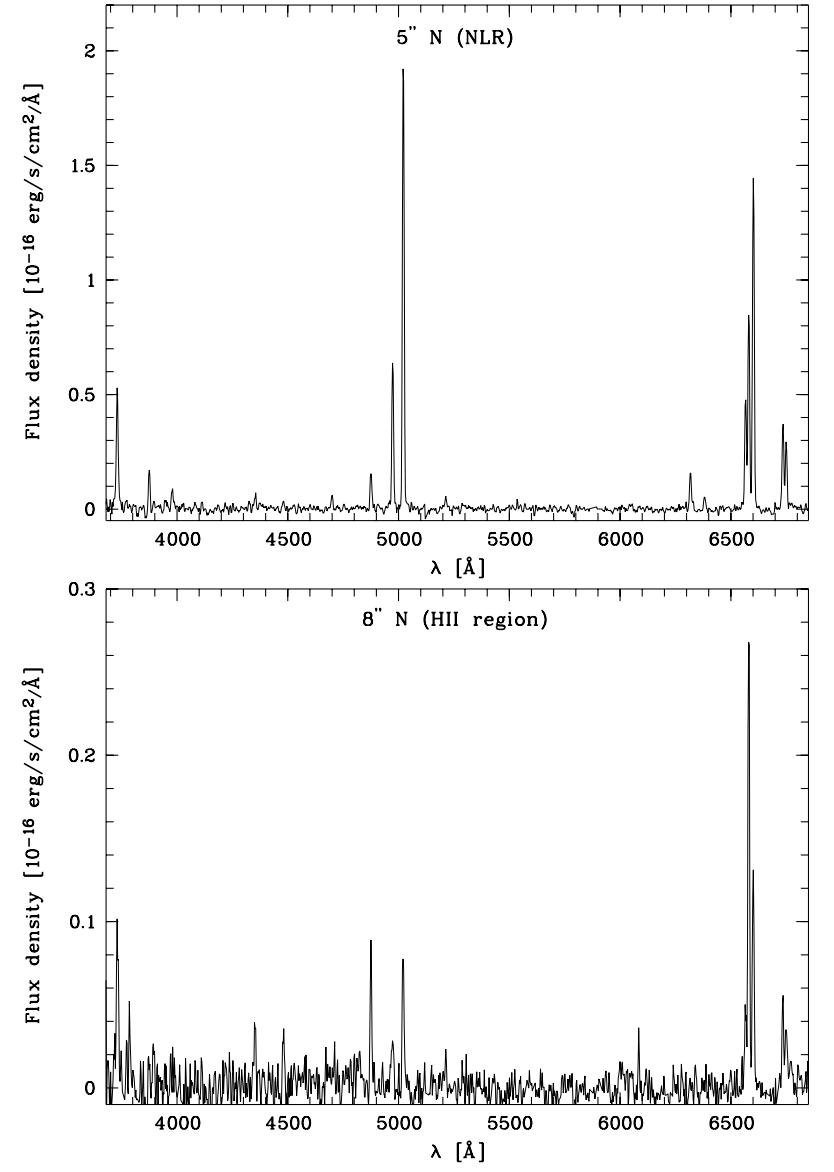

Fig. 4. Spectra of NGC 1386 at a distance of $5^{\prime \prime}$ north of the nucleus (upper panel) as well as $8^{\prime \prime}$ north of the nucleus (lower panel), respectively. While at $5^{\prime \prime}$ north of the nucleus, the lines show typical AGN ratios (i.e. $[\mathrm{O} \mathrm{III}] / \mathrm{H} \beta>3,[\mathrm{~N} \mathrm{II}] / \mathrm{H} \alpha>1$ ), at $8^{\prime \prime}$ distance, the ratios are typical for $\mathrm{H}$ II regions (i.e. $[\mathrm{O} \mathrm{III}] / \mathrm{H} \beta<3$, [N II] $/ \mathrm{H} \alpha<1$ ).

continuum slope and not an intrinsically redder stellar population in the central part.

We cannot compare the absolute values of $E_{(B-V)}$ directly as the reddening determined from the continuum slope is a value relative to the reddening of the outer region in the galaxy, i.e. the template. (For comparison, the smallest reddening correction $E_{(B-V)}$ needed to fit the continuum of the stellar template to that of the observed central spectra was set arbitrarily to zero in Fig. 5, upper panel.) However, the reddening value obtained from the emission-line ratio $\mathrm{H} \alpha / \mathrm{H} \beta$ does not show a comparable regular distribution. Moreover, the reddening of the continuum slope covers a range of $\sim 0.3$ in $E_{(B-V)}$, while a three times larger range is covered in the reddening distribution derived from the Balmer decrement $\left(\Delta E_{(B-V)} \sim 1\right)$. This can be due to extinction by foreground dust in e.g. the host galaxy which affects both the template and the central spectra and thus do not reflect in the relative reddening value determined from the difference in continuum slope of the template and the NLR spectra.

It seems that the stellar population and the NLR are suffering different dust extinctions. Possible explanations are (i) high density leading to an additional collisional contribution to the observed $\mathrm{H} \alpha / \mathrm{H} \beta$ ratio independent from dust. However, 

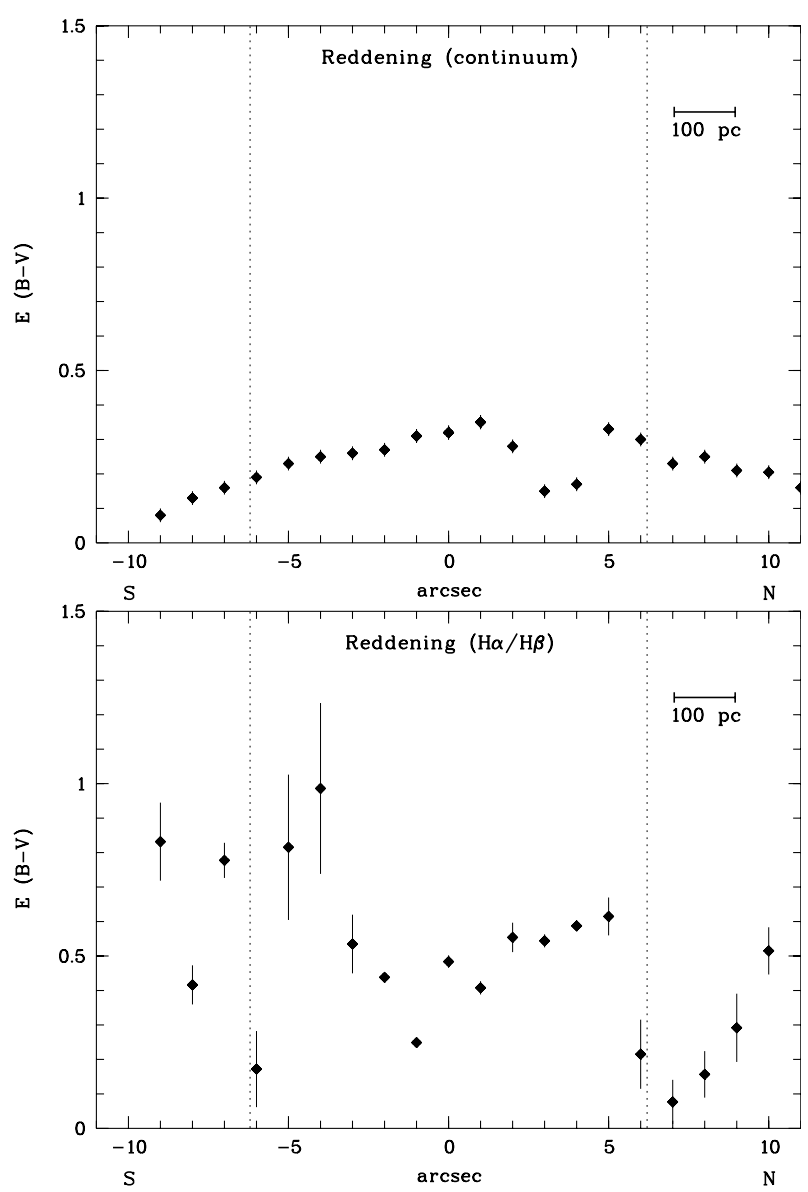

Fig. 5. Upper panel: reddening distribution of the continuum of the central spectra relative to that of the stellar template. Lower panel: reddening distribution derived from the recombination value $\mathrm{H} \alpha / \mathrm{H} \beta$. In both panels, the edge of the NLR as determined from the diagnostic diagrams is indicated by dotted lines.

extremely high densities are needed to explain the large ratios and there is no observational evidence for such high densities. (ii) Patchy dust clouds beyond the NLR may absorb the emission lines along the line-of-sight of the NLR clouds. Also this explanation is rather unlikely. We favor scenario (iii): the dust is intrinsic to the NLR clouds and its column density varies along the line of sight, resulting in the observed differences in the reddening of the NLR and the stellar template.

We use the reddening distribution determined from the $\mathrm{H} \alpha / \mathrm{H} \beta$ emission-line ratio to correct for the intrinsic reddening of the NLR itself as these lines originate in the NLR and thus give a better estimate for the reddening within the NLR than the one determined from the continuum slope.

\subsection{Spatially resolved spectral diagnostics}

Diagnostic line-ratio diagrams of the type pioneered by Baldwin et al. (1981) are commonly used to distinguish between emission-line object classes (e.g. Seyfert galaxies, LINERs, Starbursts, transition objects), referring to a "total" spectrum or the central spectrum of one object. Here, we make use of the spatially resolved spectra to discriminate between different regions in the same object, for example the NLR and
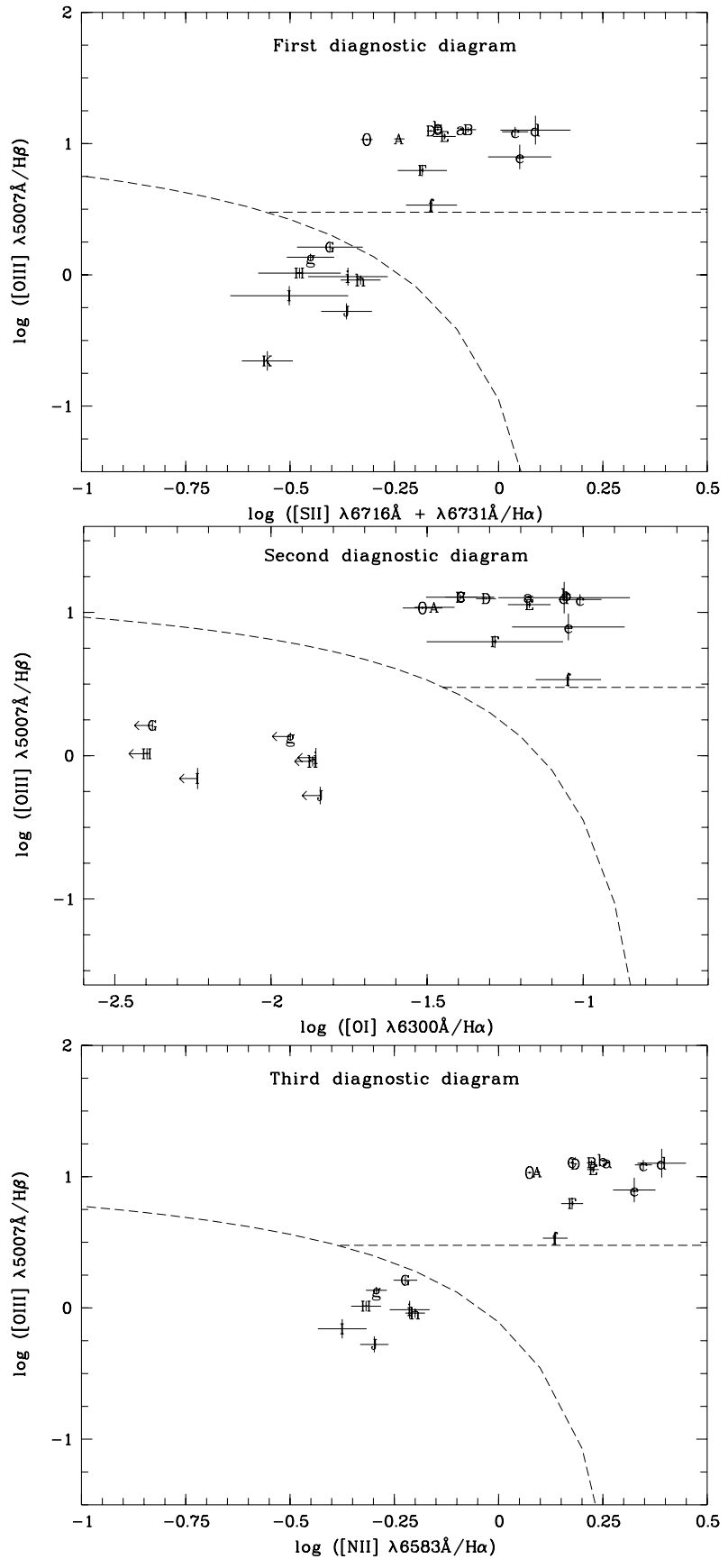

Fig. 6. Diagnostic diagrams for spatially resolved line ratios in NGC 1386. The dividing lines were taken from the analytic AGN diagnostics of Kewley et al. (2001). The symbols are chosen such that "0" refers to the central spectrum, the small letters mark southern regions, the capital ones northern regions ("a,A" $=1$ " distance from the nucleus $=52 \mathrm{pc}, " \mathrm{~b}, \mathrm{~B} "=2$ " distance $=104 \mathrm{pc}$ etc.). In all three diagrams, only the central $r \sim 6^{\prime \prime}$ (310 pc; A,a - F,f) show ratios expected for AGNphotoionised gas (upper right corner). Further out (until $r \sim 10^{\prime \prime} ; \mathrm{G}, \mathrm{g}$ - J,i), the line ratios fall into the $\mathrm{H}$ II-region regime (lower left corner).

circumnuclear or extended HII regions. Thus, to probe the "real" NLR size, i.e. the central region which is photoionised by the AGN, and to discriminate the contribution from circumnuclear starbursts, we determined spatially resolved emissionline ratios. 

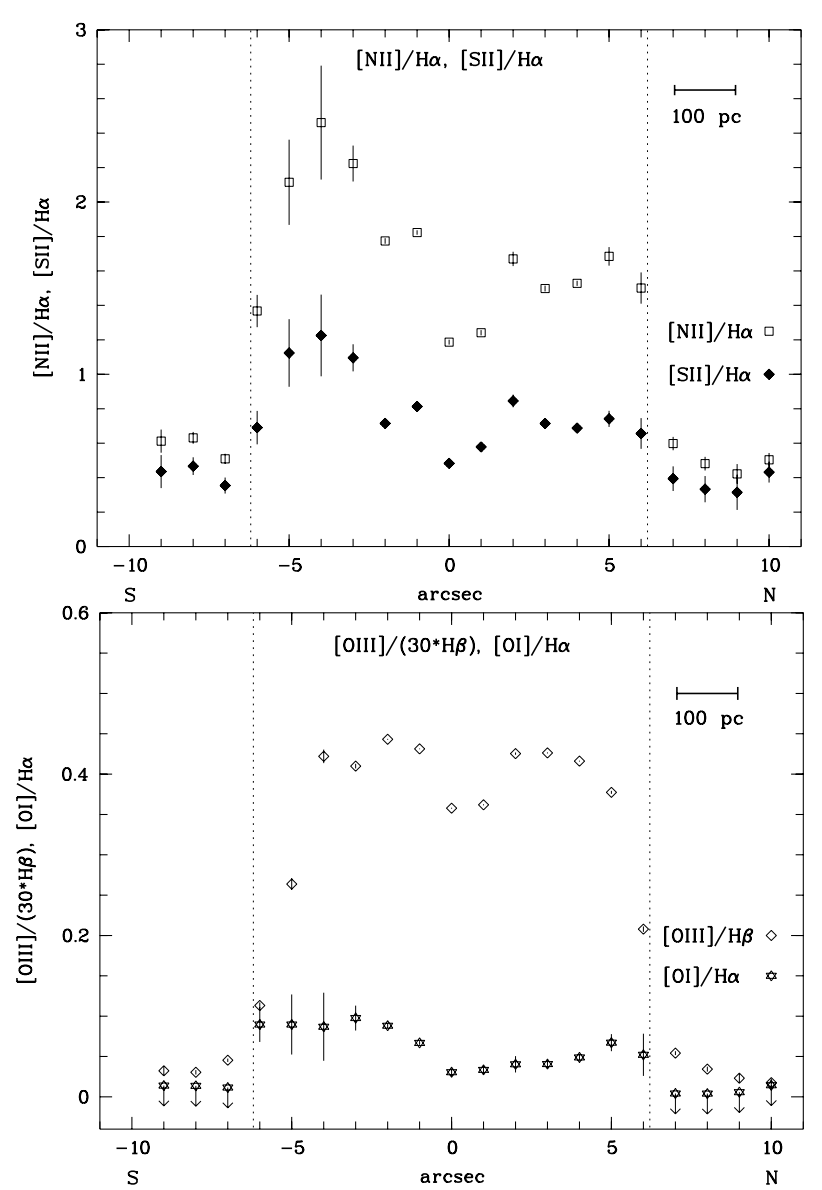

Fig. 7. Reddening corrected emission-line ratios used in the diagnostic diagrams as a function of distance from the nucleus: $[\mathrm{N} \mathrm{II}] / \mathrm{H} \alpha$ and $[\mathrm{S} \mathrm{II}] / \mathrm{H} \alpha$ (upper panel) as well as $[\mathrm{O} \mathrm{III}] / \mathrm{H} \beta$ and $[\mathrm{O} \mathrm{I}] / \mathrm{H} \alpha$ (lower panel). Note that the $[\mathrm{O} \mathrm{III}] / \mathrm{H} \beta$ ratio was divided by a factor of 30 for comparison. The edge of the NLR as determined from the diagnostic diagrams is indicated by dotted lines.

In Fig. 6, we present three diagnostic diagrams of NGC 1386. The high $\mathrm{S} / \mathrm{N}$ ratio of our spectra enables us to measure line ratios for all three diagrams out to $10^{\prime \prime}$ from the nucleus. The symbols are chosen such that " 0 " refers to the central spectrum, the small letters mark southern regions, the capital ones northern regions ("a, $A$ " $=1$ " distance from the nucleus $=52 \mathrm{pc}$, "b,B" $=2$ " distance $=104 \mathrm{pc}$ etc. .). In the second diagnostic diagram, the data points of the outer $7-10^{\prime \prime}$ are upper limits, due to the faintness of the [O I] $\lambda 6300 \AA$ line (hereafter $[\mathrm{O} \mathrm{I}]$ ) involved. The line ratios of the central regions up to $6 "$ lie in the AGN regime $(0, \mathrm{~A} / \mathrm{a}-\mathrm{F} / \mathrm{f})$. The outer line ratios extending to $10^{\prime \prime}(\mathrm{G} / \mathrm{g}-\mathrm{J} / \mathrm{i})$ all lie in the lower left corner usually covered by $\mathrm{H}$ II regions. This is a rather sharp transition which can be interpreted as the edge of the AGN photoionised region, i.e. the NLR, occuring at a radius of $6^{\prime \prime}(310 \mathrm{pc})$, while emission extending to $10^{\prime \prime}$ originates from circumnuclear H II regions. These $\mathrm{H}$ II regions can be attributed to the $\mathrm{H}$ II regions seen by Tsvetanov \& Petrosian (1995) in their $\mathrm{H} \alpha+[\mathrm{N} \mathrm{II}]$ image, occuring at distances of $\sim 6-11^{\prime \prime}$ from the nucleus along a p.a. of $5^{\circ}$ as used in our observations.

Our results agree with that of Weaver et al. (1991) who report an NLR extension of $\sim 6^{\prime \prime}$ based on the third diagnostic diagram. In the first and second diagnostic diagram, their data only include the nuclear region due to the faintness of the involved [OI] and [S II] lines in the outer regions. In their third diagnostic diagram, Weaver et al. (1991) find some evidence for high $[\mathrm{N} \mathrm{III}] / \mathrm{H} \alpha$ plus low $[\mathrm{O} \mathrm{III}] / \mathrm{H} \beta$ ratios typical for LINERs surrounding the highest ionisation gas near the nucleus. Our data do not show LINER-type ratios. The discrepancy between the two results may be due to different stellar templates used. The influence of the stellar template is discussed in Appendix A.

In Fig. 7, we show the strongest reddening-corrected line ratios as a function of radius.

Compared to pure imaging, using diagnostic diagrams to determine the NLR size has the advantage to be less sensitive to flux depth and to exclude contributions of circumnuclear starbursts. Comparing the NLR size (radius $6^{\prime \prime} \simeq 310 \mathrm{pc}$ ) determined from our diagnostic diagrams with literature values, we find that it is twice as large as the one found by Schmitt et al. (2003a) (3", Fig. 1), possibly due to the low sensitivity of the HST snapshot survey (short integration time of $800 \mathrm{~s}$ using the $2.4 \mathrm{~m}$ HST mirror compared to $1800 \mathrm{~s}$ with the $8 \mathrm{~m}$ VLT mirror and a 20 times larger pixel size ${ }^{5}$ ). Fraquelli et al. (2003) detect line emission which they classify as extended NLR out to a distance of $\sim 10^{\prime \prime}$ from the nucleus. However, our analysis shows that the extended emission between $6^{\prime \prime}$ and $10^{\prime \prime}(310-520 \mathrm{pc})$ originates from circumnuclear H II regions and cannot be attributed to the NLR. Note that the total [O III] emission with a $S / N>3$ in our spectra extends out to $r \sim 12^{\prime \prime}$, but only the central $r \sim 6^{\prime \prime}$ can be attributed to the NLR.

As already pointed out by Weaver et al. (1991), the sharp transition can be interpreted as a real physical transition between the AGN powered NLR and the surrounding H II regions in the disk. H II regions may be present over the entire emission-line region but inside a distance of $6^{\prime \prime}$, the AGN ionisation dominates. Therefore, it is reasonable to define the radius determined from the transition seen in the diagnostic diagrams as the size of the NLR. This conclusion is further supported by photoionisation models presented in the following section.

\subsection{NLR size versus photoionisation modeling}

The determination of the NLR size depends on the definition of the NLR itself. We here assume that the NLR consists of gas photoionised by the central AGN. Thus, we are able to determine the NLR size from the observed transition of line ratios in the diagnostic diagrams from the AGN regime to that typical for $\mathrm{H}$ II regions (Fig. 6).

We have checked whether any mechanism exists which drives line ratios in the diagnostic diagrams from the AGN regime towards and into the regime usually covered by $\mathrm{H}$ II regions, despite photoionisation by an intrinsic AGN continuum source. This question is of interest not only for the determination of the NLR radius, but also for galaxy classifications based on diagnostic diagrams in general.

\footnotetext{
5 Note, however, that the HST observations were an imaging campaign while we carried out spectroscopy, thus these values are not directly comparable.
} 
Under the assumption of a single continuum source (the central AGN) being responsible for the ionisation, the following parameters could potentially shift line ratios from AGN-towards $\mathrm{H}$ II-like in individual diagnostic diagrams: (i) extinction; (ii) ionisation parameter in combination with (iii) metallicity gradients; or (iv) electron density. We discuss each of them in turn.

(i) Extinction can be excluded to have a large effect on the observed line ratios in the diagnostic diagrams. The line ratios in all three diagnostic diagrams were chosen to minimize reddening effects by using neighbouring lines. In addition, we measured the reddening distribution directly (Fig. 5, lower panel) and use these results to correct for the reddening. Using the different reddening distribution determined by the continuum slope (Fig. 5, upper panel) does not change the results within the errors.

Models (ii)-(iv) presented below are based on the photoionisation code CLOUDY (e.g. Ferland et al. 1998) and assume a pointlike AGN continuum source which illuminates clouds of constant density. The spectral-energy distribution is a typical mean Seyfert continuum composed of piecewise power laws (Komossa \& Schulz 1997) with, in particular, an energy in$\operatorname{dex} \alpha_{\mathrm{uv}-\mathrm{x}}=-1.4$ in the EUV and a photon index $\Gamma_{\mathrm{x}}=-1.9$ in X-rays. The clouds are assumed to be ionisation bounded and of solar metallicity, unless stated otherwise. In Fig. 8, we present the effects of varying ionisation parameter (solid line 1), varying metal abundances (dotted lines 2 and 3), varying nitrogen $(\mathrm{N})$ and sulphur (S) abundances (dash-dotted lines 4 and 5), and high density (dashed line 6) on the emission-line ratios in the diagnostic diagrams.

(ii) The ionisation parameter $U$ is defined as $U=$ $Q /\left(4 \pi c n_{\mathrm{e}} r^{2}\right)$ with $Q=$ rate of H-ionising photons, $n_{\mathrm{e}}=$ electron density, and $r=$ distance between photoionising source and emission-line clouds. Variations in ionisation parameter alone have been studied by many authors and generally lead into the LINER-region of diagnostic diagrams for small ionisation parameters under the assumption of solar abundances (e.g. Stasinska 1984; Osterbrock 1989). This trend is shown in Fig. 8 (solid line 1) which includes the range of ionisation parameters we measure for NGC 1386, varying from $\log U=$ $-4.0 \ldots-1.5$ in steps of 0.5 (marked by circles) from bottom to top. Sequences were calculated three times, using densities $n=800,400$, and $200 \mathrm{~cm}^{-3}$ and $r=50,100$, and $250 \mathrm{pc}$, respectively (printed as circles from right to left; only the data points for $n=800 \mathrm{~cm}^{-3}$ and $r=50 \mathrm{pc}$ are connected by a line). We come back to (ii) in combination with ( iii $_{\mathrm{b}}$ ) below.

(iii) The strongest hint argueing against metallicity variations to change the line ratios in the observed direction is the similarity of all three diagnostic diagrams. In all three diagrams, the line ratios of each spectrum fall in the same regime, either AGN or $\mathrm{H}$ II region. In fact, while extreme parameters may shift line ratios towards $\mathrm{H}$ II-like in individual diagrams, no mechanism does so for all three, as we will show in the following.

(iii $\left.{ }_{\mathrm{a}}\right)$ Metal abundances were varied between $Z=(0.05-$ 3.0) $\times$ solar. Systematically decreasing abundances first lead to an increase in line ratios which can be partly traced back to oxygen being a strong coolant, and thus an increase of heating in case of oxygen depletion (Fig. 8).

The dotted lines 2 and 3 show models of varying metal abundances $(3,2.5,1.7,1.3,1.0,0.9,0.5,0.3,0.1,0.05 \times$ solar; from right to left) for constant ionisation parameter. The lower dotted line 2 corresponds to $\log U=-2.8$, the upper dotted line 3 to $\log U=-3.7$.

(iii ${ }_{b}$ ) If, instead, only $\mathrm{N}$ and $\mathrm{S}$ abundances are varied, line ratios shift horizontally in the [N $\mathrm{NI}]$ and [S II] diagrams towards the H II regime (Fig. 8). The dash-dotted lines 4 and 5 show models of varying $\mathrm{N}$ and $\mathrm{S}$ abundances $(3,2.5,1.7,1.3,1.0$, $0.9,0.5,0.3,0.1,0.05 \times$ solar; from right to left) for constant ionisation parameter. The upper dash-dotted line 4 corresponds to $\log U=-2.8$, the lower dash-dotted line 5 to $\log U=-3.7$.

Thus, a combination of outwards decreasing ionisation parameter and decreasing metal abundances would place line ratios in the HII-regime in the observed way, despite AGNintrinsic excitation. However, the same trend does not hold for the [OI] diagram. Just based on oxygen lines, this diagram is rather insensitive to variations in $\mathrm{N}$ and $\mathrm{S}$ abundances and line ratios remain well within the AGN regime for the whole parameter range (Fig. 8). We conclude that combined ionisation parameter and metallicity effects cannot explain the observations.

(iv) Finally, the presence of a high density component will again shift $[\mathrm{N} \mathrm{II}]$ and $[\mathrm{S} \mathrm{II}]$ towards the $\mathrm{H}$ II regime. To demonstrate an extreme, we plot in Fig. 8 the case for $\log n_{\mathrm{H}}=6$ (dashed line 6). The ionisation parameter varies from bottom to top in steps of 0.5 from $\log U=-4.0 \ldots-1.5$. Again, while [N II] and [S II] decrease, [O I] is strongly boosted, moving line ratios away from $\mathrm{H}$ II-like excitation. This effect can be traced back to the different critical densities.

We conclude that the observed distinction between $\mathrm{H}$ II-like and AGN-like line ratios of NGC 1386 represents a true difference in ionisation source, and thus our method to measure the NLR radius is valid. The second diagnostic diagram including the [O I] emission-line, new in our study compared to literature data, was essential to reach this conclusion, since our photoionisation calculations show that there are means to reach the $\mathrm{H}$ IIregime with an AGN as ionising source in the [N II] and [S II] diagrams.

\subsection{Surface-brightness distribution}

In Fig. 9, we present the surface-brightness distribution of the [O III] and $\mathrm{H} \alpha$ emission line as well as of the continuum (at 5450-5700 $)$. The surface-brightness distributions are similar to each other, centrally peaked and all decreasing with distance from the nucleus. They reveal a secondary peak $\sim 3^{\prime \prime}$ north of the nucleus and a slightly lower tertiary peak $\sim 4^{\prime \prime}$ south of the nucleus. While the [O III] surface brightness is exceeding that of $\mathrm{H} \alpha$ in the central parts, this behaviour changes at the edge of the NLR as determined from our 2D diagnostic diagrams (indicated by the dotted lines).

For comparison, the [O III] surface-brightness distribution from the HST image of Schmitt et al. (2003a) is also shown. It was derived by averaging three vectorplots along the major 

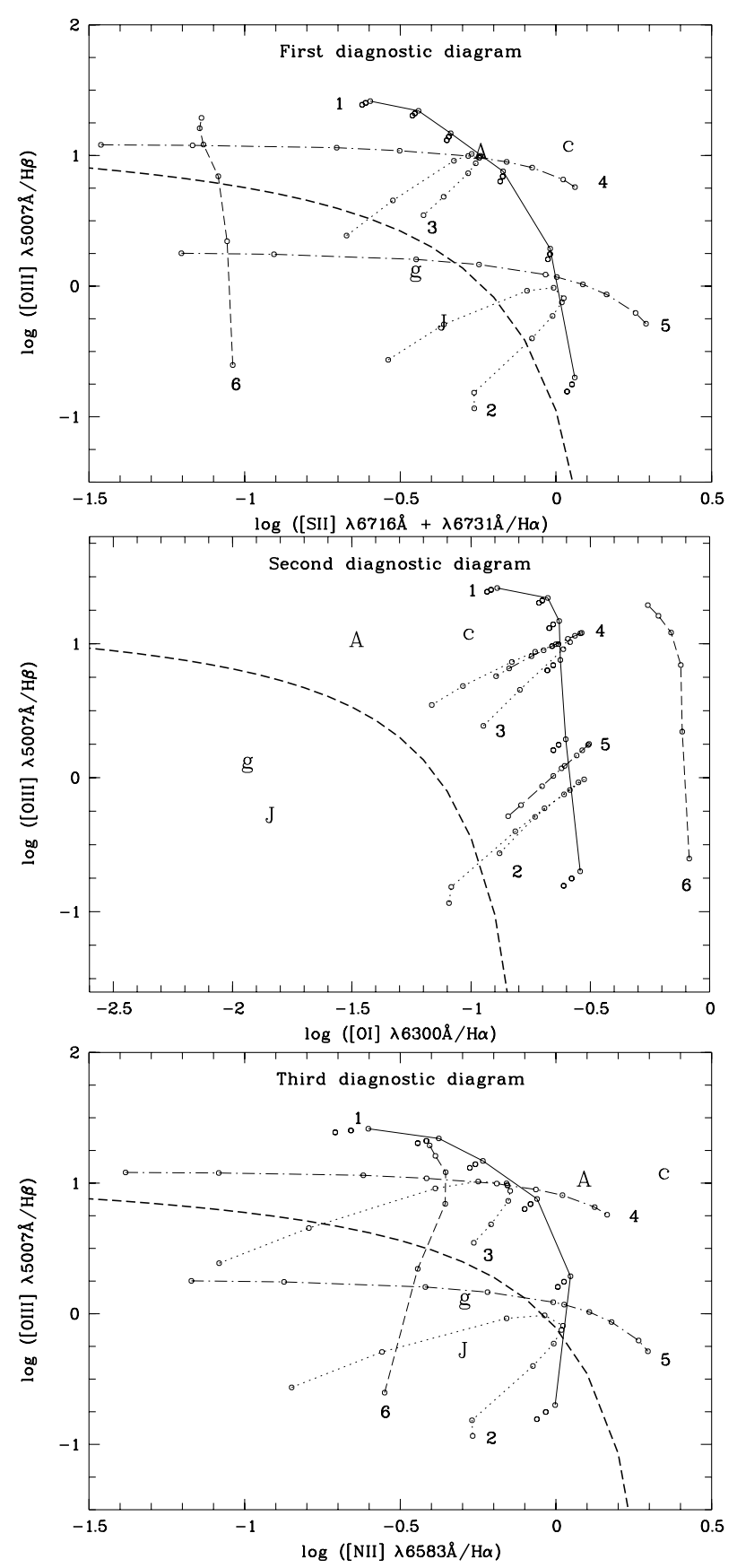

Fig. 8. CLOUDY modeling results demonstrating the effects of varying ionisation parameter (solid line 1), varying metal abundances (dotted lines 2 and 3), varying $\mathrm{N}$ and $\mathrm{S}$ abundances (dash-dotted lines 4 and 5), and high density (dashed line 6) (see text for details). The bold dashed line indicates the separation between AGN regime and $\mathrm{H}$ II regions. Four representative line ratios of NGC 1386 are also shown, falling in the $\operatorname{AGN}(\mathrm{A}, \mathrm{c})$ and $\mathrm{H}$ II-region regime $(\mathrm{J}, \mathrm{g})$.

axis of the NLR emission along PA $=5^{\circ} \pm 1^{\circ}$. It clearly shows the higher spatial resolution of the HST image $\left(0 .^{\prime} 0455\right.$ pix $\left.^{-1}\right)$ compared to the $1^{\prime \prime}$ spatial sampling of our spectral data. However, as already mentioned in Sect. 4.2, it once again reveals the low sensitivity of the HST image compared to our spectroscopy as it detects [O III] emission at a S/N of 3 only out to 3-4" from the nucleus. The HST [O III] surface-brightness

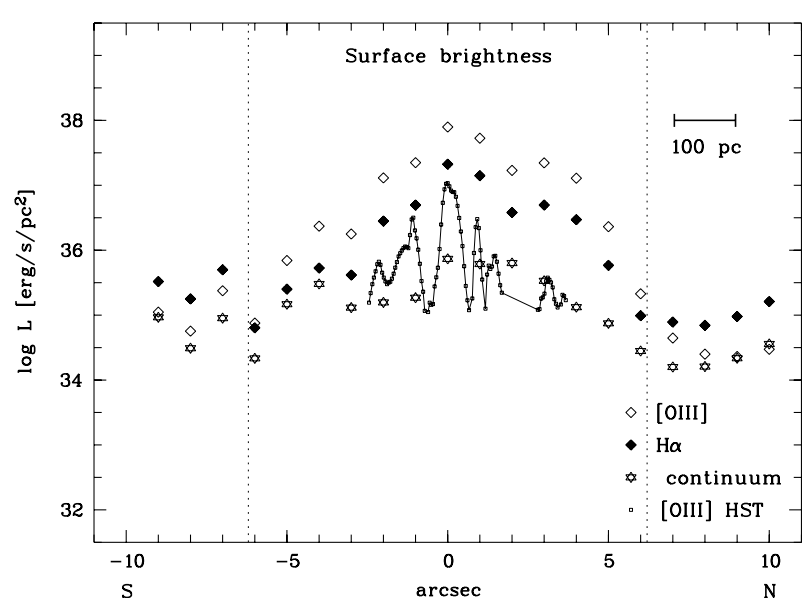

Fig. 9. Surface-brightness distribution of NGC 1386 in [O III] (open diamonds), $\mathrm{H} \alpha$ (filled diamonds), and continuum (at 5450-5700 А, stars). Error bars are smaller than the symbol size. The [O III] surfacebrightness distribution from the HST image with $S / N>3$ is shown as small open squares connected by a line. The HST image has a 20 times higher spatial resolution but a significantly lower sensitivity, not allowing to measure the outer parts of the NLR. The edge of the NLR as determined from the diagnostic diagrams is indicated by dotted lines.

distribution reveals several subpeaks of possibly individual NLR clouds, as can be already seen in the [O III] image (Fig. 1). These substructures are smoothed out in the $\sim 20$ times lower spatial resolution of our spectra.

\subsection{Electron-density distribution}

Applying the classical methods outlined in Osterbrock (1989), we derive the electron density as a function of distance from the nucleus using the ratio of the [S II] $\lambda \lambda 6716,6731 \AA$ (hereafter [S II]) pair of emission lines (Fig. 10). We used the observed central temperature to correct for the dependency of electron density on temperature ${ }^{6}\left(T_{\mathrm{e}} \simeq 15650 \mathrm{~K}\right)$. Due to the faintness of the involved [O III] $\lambda 4363 \AA$ emission line, we were not able to measure the temperature in the outer parts.

The electron density is highest at the nucleus with $n_{\mathrm{e}} \simeq$ $1540 \mathrm{~cm}^{-3}$ and decreases outwards down to the low-density limit (assumed to be $50 \mathrm{~cm}^{-3}$ ). A secondary peak can be seen at a distance of $6^{\prime \prime}$ to the north of the nucleus $\left(n_{\mathrm{e}} \simeq 670 \mathrm{~cm}^{-3}\right){ }^{7}$

Comparing the position of the observed northern $n_{\mathrm{e}}$ peak with the 2D electron-density distribution of Weaver et al. (1991), the closest data point is at $\sim 6$.' 5 north-east from the center at roughly a PA of $3^{\circ}$ with $n_{\mathrm{e}} \leq 100 \mathrm{~cm}^{-3}$ (their Fig. 10d). The spatial distance between this data point and our $n_{\mathrm{e}}$ peak is $\sim 25 \mathrm{pc}$. Thus, the $n_{\mathrm{e}}$ peak observed in our spectra may be attributed to an individual NLR cloud or local density inhomogeneities which our line-of-sight happens to pass. Mauder et al. (1992) also detect individual NLR clouds using speckle

\footnotetext{
${ }^{6} n_{\mathrm{e}}(T)=n_{\mathrm{e}}(\mathrm{obs}) \cdot \sqrt{(T / 10000)}$.

7 Note that the temperature can be a function of distance from the central AGN and is most probably decreasing, if the AGN is the only heating source. In such a case, correcting with the central temperature overestimates the electron density in the outer parts. Thus, a decreasing temperature can steepen the slope of the electron density.
} 


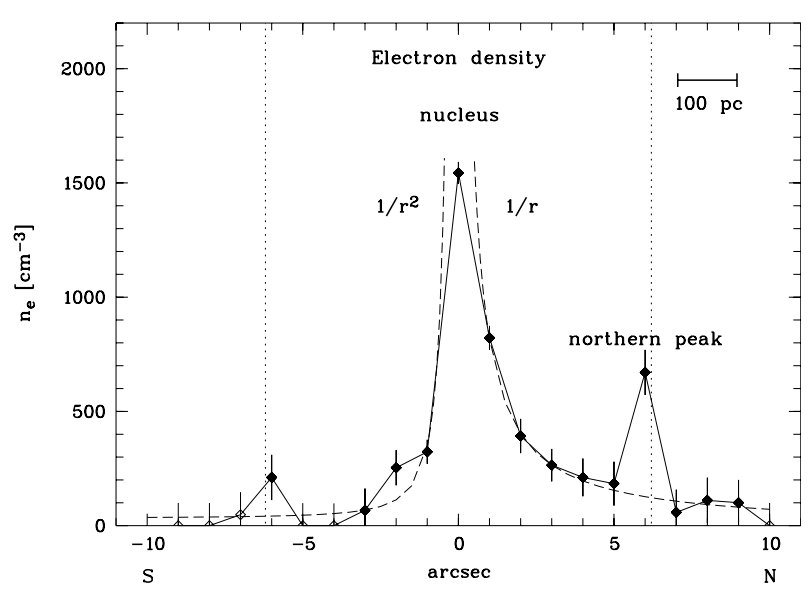

Fig. 10. Electron density obtained from the $[\mathrm{S}$ II $] \lambda 6716 \AA / \lambda 6731 \AA ̊$ ratio as a function of distance from the nucleus. Open symbols indicate locations where $n_{\mathrm{e}}$ is in the low-density limit (assumed $\leq 50 \mathrm{~cm}^{-3}$ ). The electron density decreases with radius and shows a secondary peak $6^{\prime \prime}$ north of the nucleus as well as a tertiary fainter one at $6^{\prime \prime}$ south. The fits $n_{\mathrm{e}}(r)=n_{\mathrm{e}, 0} r^{-1}$ and $n_{\mathrm{e}}(r)=n_{\mathrm{e}, 0} r^{-2}$ are shown as dashed lines. The edge of the NLR as determined from the diagnostic diagrams is indicated by dotted lines.

interferometry and conclude that the ionising radiation must be absorbed on scales $<15 \mathrm{pc}$ in a clumpy structure of the NLR. Interestingly, the peak occurs at the edge of the NLR. We discuss the origin of the northern peak in greater detail in Section 4.7. Another, but significantly smaller peak is visible at the southern edge $\left(n_{\mathrm{e}} \simeq 210 \mathrm{~cm}^{-3}\right)$.

While a detailed fit of a power-law function is complicated by the intrinsic scatter of the data points, a general dependency on $r$ can be estimated. The best fits of a power-law function with $n_{\mathrm{e}}(r)=n_{\mathrm{e}, 0} r^{-\delta}$ were derived with $\delta$ between 1 and 2 for the electron density (Fig. 10), neglecting the data point $6^{\prime \prime}$ north of the nucleus which may have another origin (Sect. 4.7).

\subsection{Ionisation-parameter distribution}

To estimate the value of the ionisation parameter, the line ratio [O II] $\lambda 3727 \AA /[\mathrm{O}$ III] $\lambda 5007 \AA$ (hereafter [O II]/[O III]) can be used [e.g. Penston et al. (1990)]. Komossa \& Schulz (1997) have shown that in case of low density $\left(n_{\mathrm{H}}<10^{3} \mathrm{~cm}^{-3}\right)$, this line ratio yields $U$ independent of the shape of the ionising continuum. It gives a good approximation of the ionisation parameter as long as there are no strong density inhomogeneities with radius. If the NLR contains a mixture of densities, the ratio predicts a too high value of $U$. But even if the absolute value of $U$ was overpredicted, the observed slope would not change. We used the theoretical variations of [O II $] /[\mathrm{O}$ III] calculated by Komossa \& Schulz (1997) (their Fig. 7) with $U$ for a powerlaw continuum $\left(\alpha_{\mathrm{uv}-\mathrm{x}}=-1.5\right)$ and a density of $2<\log n_{\mathrm{H}}$ $\left(\mathrm{cm}^{-3}\right)<3$ (Fig. 11). As the electron density is decreasing with distance from the nucleus ( 1500 to $\sim 50 \mathrm{~cm}^{-3}$, Fig. 10), the resulting ionisation parameter lies in between the two curves calculated for $\log n_{\mathrm{H}}=2$ and $\log n_{\mathrm{H}}=3$, i.e. the slope narrows.

While Weaver et al. (1991) propose a decreasing ionisation parameter to explain the observed line ratios in NGC 1386, we

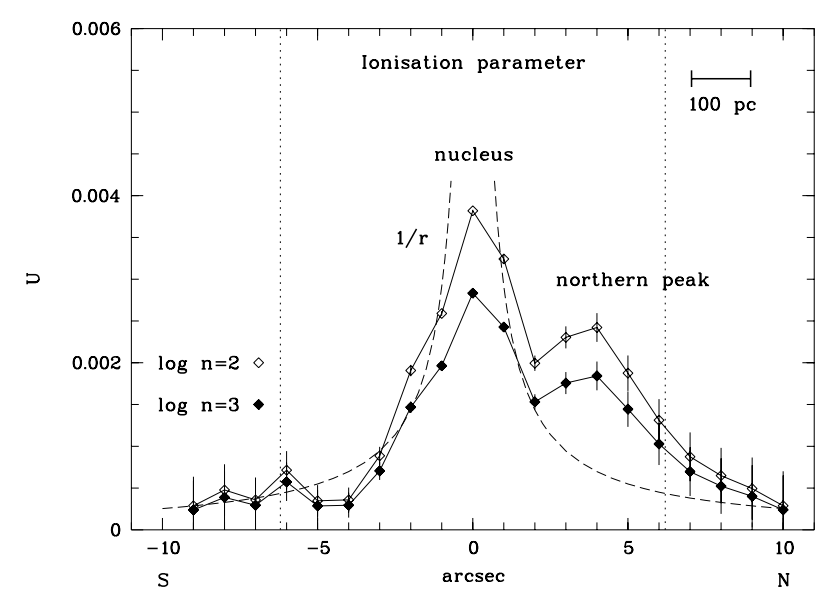

Fig. 11. Ionisation parameter derived from $[\mathrm{O}$ II $] /[\mathrm{O}$ III $]$ ratio as a function of distance from the nucleus (open symbols: $n_{\mathrm{H}}=100 \mathrm{~cm}^{-3}$, filled ones: $n_{\mathrm{H}}=1000 \mathrm{~cm}^{-3}$ ). The ionisation parameter is highest in the center and decreases with distance with a secondary peak at $4^{\prime \prime}$ to the north of the center. The fit $U(r)=U_{0} r^{-1}$ is shown as dashed line. The edge of the NLR as determined from the diagnostic diagrams is indicated by dotted lines.

can determine the ionisation parameter directly and indeed find that it is decreasing with distance from the nucleus. It varies between $U_{\log \left(n_{\mathrm{e}}\right)=3}=(2.83 \pm 0.01) \times 10^{-3}$ and $2.4 \times 10^{-4}$ within the NLR with the highest value in the center. It reveals a secondary peak at 4 " to the north of the center which is at the north edge of a "blob" of emission clearly visible in the [O III] image from Schmitt et al. (2003a) (Fig. 1). Compared to the electron density, the secondary peak is $2^{\prime \prime}$ closer to the nucleus and significantly broader. We discuss the origin in Sect. 4.7. In Appendix B, we discuss any possible influence of the reddening distribution on the slope of the ionisation parameter.

We estimated the general dependency of the decrease of $U$ on $r$. The best fit of a power-law function with $U(r)=U_{0} r^{-\delta}$ is derived with $\delta \sim 1$ (Fig. 11), again neglecting the "northern peak". Inserting the fit $n_{\mathrm{e}} \propto r^{-1}$ to the definition of $U=$ $Q /\left(4 \pi c n_{\mathrm{e}} r^{2}\right)$ yields $U \propto r^{-1}$. This is in agreement with the fit to the ionisation parameter. It indicates that the excitation of the bulk of the NLR is due to photoionisation and that ionisation by e.g. shocks is negligible.

\subsection{Northern peak: evidence for shocks}

Our observations show that both the electron density and the ionisation parameter are decreasing with radius. They show a secondary peak $\sim 6^{\prime \prime}$ and $4^{\prime \prime}$ north of the center, respectively. The peak at $4^{\prime \prime}$ is close to the northern tip of the [O III] emission (Fig. 1). In the same region, a blue continuum indicating low extinction is observed (Fig. 5, upper panel, see also Ferruit et al. 2000).

Can both the increased density and the ionisation parameter originate from a shock of a radio jet? Extended radio emission has been found by Nagar et al. (1999), displaced to the observed slit PA tracing the major [O III] extension by $\sim 15^{\circ}\left(\mathrm{PA}_{\text {radio }}=170^{\circ}\right.$ versus $\left.\mathrm{PA}_{[\mathrm{OIII}]}=5^{\circ}\right)$. A scenario in which the outflowing material causing the radio emission compresses 


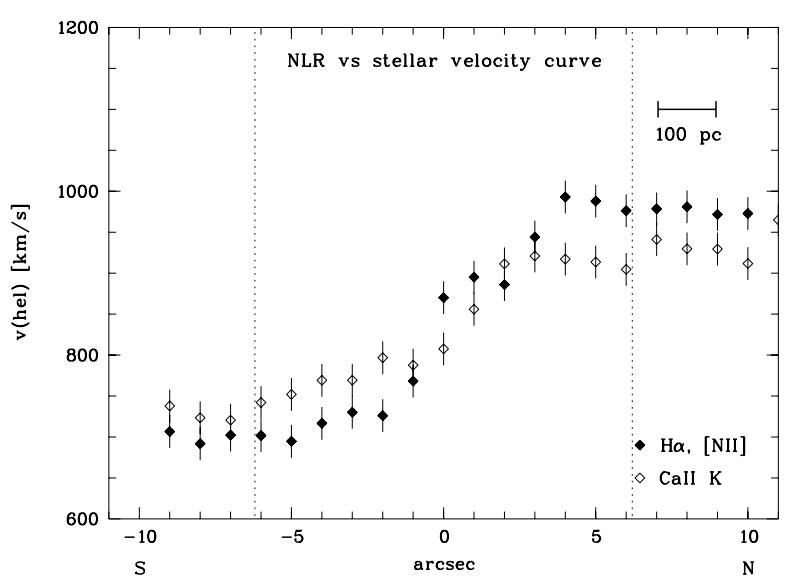

Fig. 12. Velocity curve of the NLR derived from the average value of the peak wavelengths of the $\mathrm{H} \alpha$ and [N II] emission lines (filled symbols) as well as the stellar velocity curve measured using the Ca II $\mathrm{K}$ absorption-line "peak" wavelength as seen in the "raw" spectrum (open symbols). The edge of the NLR as determined from the diagnostic diagrams is indicated by dotted lines.

the line-emitting gas by shocks, increasing the ambient density, seems to be rather common for Seyfert galaxies (e.g. Capetti et al. 1996; Falcke et al. 1998). While the shock front results in a locally increased density, the continuum of the shock can possibly ionise a larger area of the surrounding medium and produce a change in the observed line ratios, resulting in an $U$ bump which is broader than the sharp peak of $n_{e}$. Fast autoionising shocks can produce such a local continuum ionising the surrounding medium (Dopita \& Sutherland 1996).

To check the shock scenario, we searched for highionisation emission lines (e.g. $[\mathrm{Fe} \mathrm{X}] \lambda 6375 \AA$ ). In the nuclear spectra, we find strong [Fe VII $]$ 15721 $\AA$ and [Fe VII] $\lambda 6087 \AA$ emission, comparable to that of [O I]. Due to our low spectral resolution, $[\mathrm{FeX}] \lambda 6375 \AA$ is blended with the $[\mathrm{OI}] \lambda 6363 \AA$ line. Thus, to estimate the contribution of $[\mathrm{FeX}] \lambda 6375 \AA$, we fit two Gaussians to [O I] $\lambda \lambda 6300,6363 \AA$ with line fluxes fixed to the ratio of $1: 3$ and a third one to fit any remaining flux of $[\mathrm{FeX}] \lambda 6375 \AA$. Indeed, we find evidence for $[\mathrm{FeX}]$ contribution in the central $2^{\prime \prime}\left([\mathrm{FeX}] / \mathrm{H} \beta \sim 0.047\right.$ in the center, $[\mathrm{Fe} \mathrm{X}] / \mathrm{H} \beta \sim 0.049$ at $1^{\prime \prime}$ to the north) and at $6^{\prime \prime}([\mathrm{Fe} \mathrm{X}] / \mathrm{H} \beta \sim 0.049)$, i.e. at the same region where we observe an increased electron density.

The observed minimum in the reddening of the continuum slope roughly 3-4" north of the nucleus (Fig. 5, upper panel) could be mimicked by an additional local continuum contribution. The extinction in the NLR measured from the $\mathrm{H} \alpha / \mathrm{H} \beta$ value reaches a minimum at 6-7" north of the nucleus (Fig. 5, lower panel). This can be explained by the destruction of dust by local shocks.

To conclude, we find several signs for shocks. A radio jet interacting with the NLR gas is a plausible explanation for the northern peak observed in the electron density and the ionisation-parameter distribution.

\subsection{NLR versus stellar velocity curve}

We derived the NLR velocity curve from the average of the peak wavelengths of the $\mathrm{H} \alpha$ and [N II] emission lines. In addition, given the high $\mathrm{S} / \mathrm{N}$ ratio of our spectra, we are able to trace the velocity field from the "peak wavelengths" of the stellar absorption line Ca II K (before subtraction of the stellar template) throughout the whole region as this line is not blended with emission lines. The uncertainty in velocity values obtained by comparing repeated measurements at the same point is $20 \mathrm{~km} \mathrm{~s}^{-1}$. Note that gas velocities derived from the emission of [O II], $\mathrm{H} \beta$, and [O III] reveal a curve similar to that of $\mathrm{H} \alpha$, [N II].

Both, the stellar and the gaseous velocity curves, have a similar slope (Fig. 12). The stellar velocity curve covers an overall smaller range of $(\sim 720-940) \pm 20 \mathrm{~km} \mathrm{~s}^{-1}$, while the emission lines show velocities ranging from $(\sim 690-990) \pm$ $20 \mathrm{~km} \mathrm{~s}^{-1}$. We define the kinematical center by taking the symmetry center of the outer portions of the velocity curve. The kinematical center has an heliocentric velocity of $\sim 825 \pm$ $20 \mathrm{~km} \mathrm{~s}^{-1}$, i.e. slightly lower than the one given in $\operatorname{NED}\left(v_{\text {hel }}=\right.$ $868 \mathrm{~km} \mathrm{~s}^{-1}$ ). However, the velocity measured at the position of the optical nucleus is with $v_{\text {hel }}=870 \pm 20 \mathrm{~km} \mathrm{~s}^{-1}$ the same as the literature value within the errors.

The kinematical center of the stellar velocity curve lies $\sim 1$ " north of the optical nucleus ("0" on the spatial scale). This offset is in agreement with the analysis of the $\mathrm{H} \alpha$ peak velocity field by Weaver et al. (1991) and Schulz \& Henkel (2003) who report an offset of the kinematical center of $\sim 1^{\prime \prime}$ to the north-east of the photometrical center. The kinematical center is suggestive of a hidden nucleus residing 1 " north of the optical nucleus as has also been suggested by Rossa et al. (2000) (see Fig. 1).

However, the kinematical center of the NLR velocity curve we find at a PA of $5^{\circ}$ (compared to $23^{\circ}$ of Schulz \& Henkel 2003 ) is $\sim 0.5$ south of the optical nucleus. It may be indicative of a disturbed velocity field from outflows and shocks at our observed slit orientation.

While the kinematics of the emission-line region in NGC 1386 has been investigated by several authors, an interpretation of the observed velocity field is not unambiguous. Though the natural explanation is that of a slightly projected rotation curve from an inclined emission-line disk in the center, an interpretation by a bipolar flow accidentally aligned with the kinematical major axis of the rotating disk is also possible (Schulz \& Henkel 2003). However, as the velocity curves of both NLR gas and stars are similar, a bipolar outflow of the NLR is rather unlikely as it had to be decoupled from the stellar gravitational field and its accordingly stratified interstellar medium.

Instead, the NLR seems to follow the stellar rotation indicating that the NLR gas is distributed in a disk rather than a sphere. Galactic rotation of narrow-line gas seems not to be uncommon (e.g. Schulz \& Henkel 2003). Mulchaey et al. (1996b) propose that the NLR gas is distributed in the galactic disk rather than a sphere, to explain differences of the observed NLR structure with that expected from the Unified Model in the case of a spherical distribution of the NLR gas. 
The gaseous velocity curve does not show a different behaviour in the NLR and the H II-region regime. This indicates that the NLR does not consist of an intrinsically different gas component with its own kinematics, but is ambient gas in the galactic disk photoionised by the AGN.

Our results reveal some differences between the stellar and the NLR velocities in NGC 1386. The NLR velocities are in general higher than those of the stellar absorption lines. The difference is especially large in both the outer southern and northern part of the NLR. These can be due to many effects, e.g. rotating gas disk/ring within a kinematically hotter stellar component (the interpretation being complicated by the fact that due to the high inclination of the galaxy, the line of sight towards NLR intersects both the stellar disk and the bulge), gas moving along elliptical streamlines in a barred potential (keep in mind the ambiguous Hubble-type classification, Sect. 1.2), or outflow/jet interaction. However, for a detailed interpretation of the stellar and gaseous velocity field, kinematical modelling is required which lies beyond the scope of this paper.

\subsection{NLR size-luminosity relation}

The NLR size and its relation to [O III] luminosity has been studied by Bennert et al. (2002) and Schmitt et al. (2003b) based on [O III] images. While Bennert et al. (2002) find a relation between NLR size and luminosity with a slope of 0.5 , Schmitt et al. (2003b) report a slope of 0.33. If the NLRs were ambient circumnuclear gas fully photoionised by the nuclear continuum source, a Strömgren-type radius-luminosity relationship $R \propto L^{0.33} n^{-0.66}$ and thus a slope of 0.33 is indeed expected in the case of a constant density. However, a decreasing density as observed for NGC 1386 seems to be common to several Seyfert galaxies (e.g. Storchi-Bergmann et al. 1992; Fraquelli et al. 2000, 2003), contradicting the simple "Strömgren explanation".

A slope of 0.5 found by Bennert et al. (2002) for the NLR size-luminosity relation of both the [O III] emission line as well as $\mathrm{H} \beta$ is expected if on average, all AGNs have the same ionisation parameter, density, and ionising spectral energy distribution (Netzer 1990; Netzer et al. 2004). It suggests a selfregulating mechanism that determines the size of the NLR to scale rather with the ionisation parameter $U: U \propto Q /\left(R^{2} n\right)$ with $Q \propto L(\mathrm{H} \beta)$ which leads to $R \propto L(\mathrm{H} \beta)^{1 / 2}$, for a given $U$ and $n_{\mathrm{e}}$, in agreement with the empirical relation (assuming that $L(\mathrm{H} \beta) \propto L([\mathrm{OIII}]))$. At the outskirts of the NLR, $n_{\mathrm{e}} \sim 10^{2-3} \mathrm{~cm}^{-3}$ and $U \sim 10^{-(2-3)}$, so that efficient [O III] emission comes from regions with $U n_{\mathrm{e}} \sim 1$. The NLR sizeluminosity relation indicates that all objects have a similar $U n_{\mathrm{e}}$ value at the edge of their NLR, independent of the individual spatial behaviour of $U$ and $n_{\mathrm{e}}$. Thus, the observed spatially decreasing ionisation parameter and density in NGC 1386 do not contradict the explanation of the NLR size-luminosity relation in terms of a constant (in all objects) ionisation parameter.

However, the NLR size determination using [O III] images alone bears various uncertainties due to e.g. the sensitivity dependency of the observation and possible contributions from circumnuclear starbursts as has been shown for NGC 1386.
Compared to the spatially resolved spectral diagnostics measuring the "real" NLR size, the apparent NLR size determined by [O III] images can be either smaller in case of low sensitivity or larger in case of contributions of circumnuclear starbursts. Using the methods described here to determine the NLR sizes of a larger sample of objects will help to scrutinise the NLR size-luminosity relation. Nevertheless, individually differing NLR sizes may not change the overall slope of the sizeluminosity relation which extends over four orders of magnitudes in luminosity and two orders of magnitudes in size.

\section{Conclusions}

Applying diagnostic diagrams to our spatially resolved spectra, we observe a transition of emission-line ratios from the central AGN region to the surrounding $\mathrm{H}$ II regions. This transition occurs at $r \sim 6^{\prime \prime}(310 \mathrm{pc})$ to the north and south of the optical nucleus and is, for the first time, observed in all three diagnostic diagrams, i.e. including the second diagnostic diagram involving the $[\mathrm{O} \mathrm{I}]$ emission line. The most probable explanation for this transition is that the stellar ionisation field dominates that of the AGN at a distance of $\geq 6^{\prime \prime}$. We can exclude other effects such as the variation of physical parameters from CLOUDY photoionisation modelling. The second diagnostic diagram $([\mathrm{OIII}] / \mathrm{H} \beta$ versus $[\mathrm{OI}] / \mathrm{H} \alpha)$ was essential to draw this conclusion as our photoionisation calculations show that there are means to reach the H II-region regime with an AGN as ionising source in the $[\mathrm{N}$ II] and [S II] diagrams. We determine the radius of the NLR in NGC 1386 to $6^{\prime \prime}(310 \mathrm{pc})$, independent of sensitivity and excluding [O III] contamination from circumnuclear starbursts. Applying this method to a larger sample of Seyfert galaxies will help to scrutinise the NLR size-luminosity relation.

We derive physical parameters of the NLR in NGC 1386 such as reddening, surface brightness, electron density, and ionisation parameter as a function of distance from the nucleus. The differences between the reddening distributions determined from the continuum slope and the Balmer decrement argue in favour of dust intrinsic to the NLR clouds with varying column density along the line of sight. Both electron density and ionisation parameter decrease with radius. The decreasing electron density argues against a simple Strömgren behaviour of the NLR gas which has been suggested by the NLR sizeluminosity relation with a slope of 0.33 . The decreasing ionisation parameter in this individual object does not rule out a common effective ionisation parameter in AGNs as suggested by a slope of 0.5 of the NLR size-luminosity relation. In the outer part of the NLR, the secondary peak in the electron density and ionisation-parameter distribution are interpreted as signs of shocks, a scenario also supported by other observations.

The NLR and stellar velocity fields are similar and indicate that the NLR gas is distributed in a disk rather than a sphere. The differences between the two velocity fields can be due to several effects such as a rotating gas disk or ring, gas moving along elliptical streamlines in a barred potential or outflowing gas from a jet interaction.

The methods presented here were applied to a larger sample of Seyfert galaxies, revealing similar results to that of 
NGC 1386. A detailed discussion can be found in Bennert (2005) and will be summarised in an upcoming paper.

Acknowledgements. N.B. is grateful for financial support by the "Studienstiftung des deutschen Volkes". B.J. acknowledges the support of the Research Training Network "Euro3D-Promoting 3D Spectroscopy in Europe" (European Commission, Human Potential Network Contract No. HPRN-CT-2002-00305) and of the Czech Science Foundation (grant No. 202/01/D075). M.H. is supported by "Nordrhein-Westfälische Akademie der Wissenschaften". We thank Pierre Ferruit for providing and helping us with the fit/spec line-fitting tool and Gary Ferland for providing CLOUDY. Henrique Schmitt was so kind to provide the continuum-subtracted HST [O III] image of NGC 1386. This research has made use of the NASA/IPAC Extragalactic Database (NED), operated by the Jet Propulsion Laboratory, Caltech, under contract with the NASA.

\section{Appendix A: Influence of stellar template}

To probe the influence of the stellar template used, we carried out two approaches of (i) removing the weak emission lines of [O II], [O III], and [N II] still visible in the template and (ii) not using any template at all.

If we remove the weak emission lines in the stellar template $(\operatorname{method} i)$, the resulting line ratios are the same within the errors for most spectra, i.e. the weak emission is negligible against the strong NLR emission. The exceptions are the line ratios of two outer spectra $(\mathrm{H}, \mathrm{I})$ which are shifted towards higher $[\mathrm{N} \mathrm{II}] / \mathrm{H} \alpha$ values and now lie at the edge between H II region and LINER region. This is a possible explanation for the discrepancy between our results and that of Weaver et al. (1991) who find LINER-type line ratios in the outer region. They modified their stellar template derived from the galaxy by removing the remaining weak $[\mathrm{N}$ II] and $\mathrm{H} \alpha$ emission and replacing $\mathrm{H} \alpha$ by an artificial absorption line. This is a more physical approach than method (i), i.e. not only removing the remaining weak emission, but at the same time deepening the $\mathrm{H} \beta$ and $\mathrm{H} \alpha$ absorption trough. The true absorption will probably be underestimated due to weak emission in these lines. However, such a correction is difficult to estimate and the $\mathrm{H} \alpha$ absorption used by Weaver et al. (1991) probably still underestimates the true absorption.

We decided to not remove the weak emission: On the one hand, increasing the observed [O III] and [N II] emission by clipping the weak emission in the template and at the same time increasing the observed $\mathrm{H} \alpha$ and $\mathrm{H} \beta$ emission by deepening the absorption lines in the template will result in approximately the same line ratios and thus will not have a great influence on the diagnostic diagrams. On the other hand, the weak emission lines are attributable to ionised regions around young stars in the galaxy lying along our line-of-sight, and do not originate from the NLR emission. Thus, they truly contaminate the NLR emission and need to be subtracted.

Applying no correction for underlying absorption at all (method ii) yields extremely large $[\mathrm{O} \mathrm{III}] / \mathrm{H} \beta$ values $(\geq 25)$ near the nucleus as has already been pointed out by Weaver et al. (1991). In the outer parts, the absorption trough is clearly visible with a small $\mathrm{H} \beta$ emission peak in the center. In addition, the spectra show galaxy absorption features which accompany the

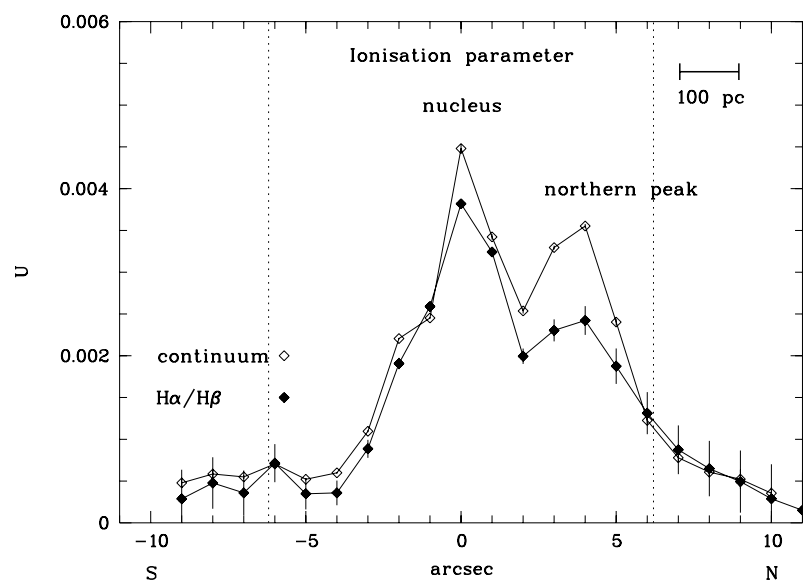

Fig. B.1. Ionisation parameter derived from $[\mathrm{O} \mathrm{II}] /[\mathrm{O} \mathrm{III}]$ ratio as a function of distance from the nucleus for $n_{\mathrm{H}}=100 \mathrm{~cm}^{-3}$. We compare the ionisation parameter derived using the reddening determined from the continuum slope for correction (open symbols) with the one from Fig. 11 using the Balmer decrement to correct for the reddening (filled symbols). The edge of the NLR as determined from the diagnostic diagrams is indicated by dotted lines.

absorption in $\mathrm{H} \beta$. These findings show the need for correcting the stellar absorption lines using a suited stellar template.

\section{Appendix B: Influence of reddening}

The only physical parameter in our study which is strongly sensitive to reddening is the ionisation parameter, derived using the line ratio $[\mathrm{O} \mathrm{II}] /[\mathrm{O} \mathrm{III}]$. We here briefly discuss the influence of the different reddening measures on the ionisation parameter.

Although we corrected for the reddening within the NLR using the recombination value $\mathrm{H} \alpha / \mathrm{H} \beta$, there is a clear difference between the reddening of the continuum slope and that determined from the Balmer decrement: While the reddening distribution determined by the ratio $\mathrm{H} \alpha / \mathrm{H} \beta$ shows a rather random distribution, the continuum slope reddening steadily increases towards the nucleus with the exception of the blue continuum at 3-4" to the north of the nucleus (Fig. 5). Can these differences explain the observed slope of the ionisation parameter, i.e. the general decrease with radius and the secondary peak at that position at which we see a blue continuum? Is it possible that we do not correct for the reddening intrinsic to the NLR gas by using the reddening value determined by recombination lines but would the continuum slope be a better tracer for the reddening distribution? To probe the difference, we used the reddening determined from the continuum slope to correct the observed [O II $] /[\mathrm{O}$ III] emission-line ratio. The resulting ionisation-parameter distribution changes only slightly showing an even steeper increase towards the center and a more pronounced secondary peak (Fig. B.1).

Thus, the observed slope in Fig. 11 is not an artefact due to a "wrong" reddening correction from the recombination value: Using the reddening determined from the continuum slope, the ionisation-parameter distribution does not flatten but, in contrary, steepen. The general conclusion of a decreasing ionisation parameter with radius which shows a secondary peak $3-4$ " north of the nucleus remains unchanged. However, we 
consider the recombination value $\mathrm{H} \alpha / \mathrm{H} \beta$ as a better indicator of the reddening distribution within the NLR as it uses emission lines originating in the NLR itself. We suggest that the differences in the reddening measurements originate from dust within the NLR with varying column density along the line of sight. Although in general using the $\mathrm{H} \alpha / \mathrm{H} \beta$ value for reddening correction bears the problem of the uncertain amount of the underlying stellar absorption, we believe that our correction using the galaxy itself as stellar template gives a good approach of the absorption-line free ratio.

\section{References}

Baldwin, J. A., Phillips, M. M., \& Terlevich, R. 1981, PASP, 93, 5

Bennert, N., Falcke, H., Schulz, H., Wilson, A. S., \& Wills, B. J. 2002 , ApJ, 574, L105

Bennert, N. 2005, Ph.D. Thesis, University of Bochum, Germany

Bica, E., \& Alloin, D. 1986, A\&A, 166, 83

Bica, E. 1988, A\&A, 195, 76

Bowen, I.S. 1960, ApJ, 132, 1

Capetti, A., Axon, D. J., Macchetto, F., Sparks, W. B., \& Boksenberg, A. 1996, ApJ, 469, 554

Cid Fernandes, R., Storchi-Bergmann, T., \& Schmitt, H. R. 1998, MNRAS, 297, 579

De Vaucouleurs, G., De Vaucouleurs, A., Corwin, H. G., et al. 1991, Third Reference Catalogue of Bright Galaxies, Vol. III (New York: Springer Verlag) (RC3)

Dopita, M. A., \& Sutherland, R. S., 1996, ApJS, 102, 161

Dopita, M. A., Groves, B. A., Sutherland, R. S., Binette, L., \& Cecil, G. 2002, ApJ, 572, 753

Falcke, H., Wilson, A. S., \& Simpson, C. 1998, ApJ, 502, 199

Ferland G. J., Korista K. T., Verner D. A., et al., 1998, PASP, 110, 761

Ferruit, P., Wilson, A. S., \& Mulchaey, J. 2000, ApJS, 128, 139

Fraquelli, H. A., Storchi-Bergmann, T., \& Binnette, L. 2000, ApJ, 532, 867
Fraquelli, H. A., Storchi-Bergmann, T., \& Levenson, N. A. 2003, MNRAS, 341, 449

Kewley, L. J., Dopita, M. A., Sutherland, R. S., Heisler, C. A., \& Trevena, J. 2001, ApJ, 556, 121

Komossa, S., \& Schulz, H. 1997, A\&A, 323, 31

Malkan, M. A., Gorjian, V., \& Tam, R. 1998, ApJS, 117, 2588

Mauder, W., Appenzeller, I., Hofmann, K.-H., et al. 1992, A\&A, 264, 9

Mulchaey, J. S., Wilson, A. S., \& Tsvetanov, Z. I. 1996a, ApJS, 102, 309

Mulchaey, J. S., Wilson, A. S., \& Tsvetanov, Z. I. 1996b, ApJ, 467, 197

Nagar, N. M., Wilson, A. S., Mulchaey, J. S., \& Gallimore, J. F. 1999, ApJS, 120, 209

Netzer, H. 1990, in Active Galactic Nuclei, ed. T. J.-L. Courvoisier, \& M. Mayor (Berlin: Springer Verlag), 57

Netzer, H., Shemmer, O., Maiolino, R., et al. 2004, ApJ, 614, 558

Osterbrock, D. E. O. 1989, Astrophysics of Gaseous Nebulae and Active Galactic Nuclei (Mill Valley: University Science Books)

Penston, M. V., Robinson, A., Alloin, D., et al. 1990, A\&A, 236, 53

Rossa, J., Dietrich, M., \& Wagner, J. S. 2000, A\&A, 362, 501

Rousset, A. 1992, PhD Thesis, Univ. J. Monnet, Saint-Étienne

Savage, B. D., \& Mathis, J. S. 1979, ARA\&A, 17, 73

Schiano, A. V. R. 1986, ApJ, 302, 81

Schlegel, D. J., Finkbeiner, D. P., \& Davis, M. 1998, ApJ, 500, 525

Schmitt, H. R., Donley, J. L., Antonucci, R. R. J., Hutchings, J. B., \& Kinney, A. L. 2003a, ApJS, 148, 327

Schmitt, H. R., Donley, J. L., Antonucci, R. R. J., et al. 2003b, ApJ, $597,768 \mathrm{~S}$

Schulz, H., \& Henkel, C. 2003, A\&A, 400, 41

Stasinska G., 1984, A\&AS, 55, 15

Storchi-Bergmann, T., Mulchaey, J. S., \& Wilson, A. S. 1992, ApJ, 395, L73

Tsvetanov, Z. I., \& Petrosian, A. R. 1995, ApJS, 101, 287

Tüg, H. 1997, ESO Messenger No. 11, p.7

Weaver, K. A., Wilson, A. S., \& Baldwin, J. A. 1991, ApJ, 366, 50 